\title{
Feedback Control of a Chaotic Finance System with Two Delays
}

\author{
Zhichao Jiang $\mathbb{D}^{1}$ and Tongqian Zhang $\mathbb{D}^{2}$ \\ ${ }^{1}$ School of Liberal Arts and Sciences, North China Institute of Aerospace Engineering, Langfang 065000, China \\ ${ }^{2}$ College of Mathematics and Systems Science, Shandong University of Science and Technology, Qingdao 266590, China \\ Correspondence should be addressed to Tongqian Zhang; zhangtongqian@sdust.edu.cn
}

Received 29 February 2020; Revised 26 April 2020; Accepted 4 May 2020; Published 26 May 2020

Academic Editor: Abdelalim Elsadany

Copyright (c) 2020 Zhichao Jiang and Tongqian Zhang. This is an open access article distributed under the Creative Commons Attribution License, which permits unrestricted use, distribution, and reproduction in any medium, provided the original work is properly cited.

\begin{abstract}
In this research, we use the double-delayed feedback control (DDFC) method in order to control chaos in a finance system. Taking delays as parameters, the dynamic behavior of the system is investigated. Firstly, we study the local stability of equilibrium and the existence of local Hopf bifurcations. It can find that the delays can make chaos disappear and generate a stable equilibrium or periodic solution, which means the effectiveness of DDFC method. By using the normal form theory and center manifold argument, one derives the explicit algorithm for determining the properties of bifurcation. In addition, we also apply some mathematical methods (stability crossing curves) to show the stability changes of the financial system in two parameters' $\left(\tau_{1}, \tau_{2}\right)$ plane. Finally, we give some numerical simulations by Matlab Microsoft to show the validity of theoretical analyses.
\end{abstract}

\section{Introduction}

In the past few decades, many scholars produced the increasing interest in nonlinear dynamic economic methods [1-11]. In the fields of finance, because of the influence of nonlinear factors, all sorts of economy problems become more and more complicated. The misalignment of certain parameters in the economic system can lead to runaway markets and possibly even a financial crisis [12-15]. Therefore, it is more and more important to study the internal structure characteristics of a complex financial system and uncover its causes, so as to predict and control the system.

A lot of work has been carried out in modeling nonlinear economic dynamics, such as Goodwin's model, van der Pol model, IS-LM model, and nonlinear finance system $[14,16-25]$. However, it is well known that even a simple nonlinear system can exhibit chaotic behavior. Chaos is the inherent randomness of deterministic systems. Since the first discovery of chaos in economics from 1985, a great impact has been produced on the study of western economics because chaos in the economic system means the inherent uncertainty in macroeconomic operation. Over the past two decades, many efforts had been made to control chaos, such as stability and chaos synchronization, at unstable fixed points. In recent years, many methods had been put forward to control and synchronize chaos, such as OGY method [26], PC method [27], fuzzy control [28], impulsive control method [29, 30], stochastic control [31-33], linear feedback control [34], delay feedback approach [35-44], and multiple delay feedback control (MDFC) [45]. Delayed feedback control (DFC) was first proposed by Pyragas [46] in order to stabilize unstable periodic orbits (UPO). Then, the DFC method was extended to the multidelay [47]. One of the main characteristics of the DFC method is that it does not need the knowledge of the internal dynamics of the system beyond the period nor does it require a preliminary understanding of the required UPO. At the same time of UPO control, using the DFC method to realize USS stability had become an area of concern and had been applied to some real systems. It is very successful in stabilizing UPO for the DFC method, but the control of USS is less efficient. In [45], authors put forward the MDFC method and conducted numerical simulations, which showed that the MDFC method preceded the DFC method in USS stability.

In [16], authors put forward a financial system describing the temporal changes using three variables: $\mathbf{x}(t)$ denotes the interest rate, $\mathbf{y}(t)$ expresses the investment demand, and $\mathbf{z}(t)$ represents the price index: 


$$
\left\{\begin{array}{l}
\dot{x}(t)=(\mathbf{y}-a) \mathbf{x}+\mathbf{z} \\
\dot{y}(t)=1-b \mathbf{y}-\mathbf{x}^{2} \\
\dot{z}(t)=-\mathbf{x}-c \mathbf{z}
\end{array}\right.
$$

where the parameters $a, b$, and $c$ represent the saving amount, the investment cost, and the elasticity of market demand, respectively, and $a, b$, and $c$ are positive constants. From [48], it is known that, under the parameter values $a=0.9, b=0.2$, and $c=1.2$, system (1) exists a strange attractor, as shown in Figure 1.

In this paper, our object is to control the strange attractor by using the DDFC method and study the following system:

$$
\left\{\begin{array}{l}
\dot{x}(t)=(\mathbf{y}-a) \mathbf{x}+\mathbf{z}+k_{1}\left[\mathbf{x}(t)-\mathbf{x}\left(t-\tau_{1}\right)\right]+k_{2}\left[\mathbf{x}(t)-\mathbf{x}\left(t-\tau_{2}\right)\right], \\
\dot{y}(t)=1-b \mathbf{y}-\mathbf{x}^{2} \\
\dot{z}(t)=-\mathbf{x}-c \mathbf{z}
\end{array}\right.
$$

where $k_{1} \in \mathbb{R}$ and $k_{2} \in \mathbb{R}$ are the feedback strengths and $\tau_{1}$ and $\tau_{2}$ are nonnegative delays.

The initial conditions of system (2) are given as

$$
\begin{aligned}
& \mathbf{x}(b)=\varphi_{1}(b), \\
& \mathbf{y}(b)=\varphi_{2}(b), \\
& \mathbf{z}(b)=\varphi_{3}(b), \quad b \in[-\tau, 0],
\end{aligned}
$$

where $\varphi=\left(\varphi_{1}, \varphi_{2}, \varphi_{3}\right)^{T} \in C=C\left([-\tau, 0], \mathbb{R}^{3}\right)$ and $\tau=\max$ $\left\{\tau_{1}, \tau_{2}\right\}$.

The purpose of this paper is to analyze and numerically study system (2). Our results show that the stability of system varies with delays. When the delay passes a certain critical value, the chaotic oscillation disappears and can be transformed into stable equilibrium or periodic orbit, which indicates that the chaotic property changes with the changes of delays.

This article is organized as follows. In Section 2, by studying the distribution of eigenvalues of exponential polynomials and using the results in [49, 50], the local stability and existence of local Hopf bifurcation are obtained. In Section 3, the properties of Hopf bifurcation are given by using central manifold theory and normal form method. In Section 4, using the crossing curve methods, it can obtain the stable changes of equilibrium in $\left(\tau_{1}, \tau_{2}\right)$ plane to overcome the problem that no information is given on the plane $\left(\tau_{1}, \tau_{2}\right)$ that comes into being stable or unstable equilibrium in Section 2. To support the analysis results, some numerical simulations are carried out in Section 5. Finally, some conclusions and discussions are given.

\section{Stability of Equilibrium and Hopf Bifurcation}

Firstly, it gives the existence of equilibria.

\section{Lemma 1.}

(i) If $c(1-a b)-b \leq 0$ holds, then system (2) has only $a$ boundary equilibrium $E_{0}(0,1 / b, 0)$ (ii) If $c(1-a b)-b>0$ holds, then system (2) has two interior equilibria $E_{+}^{*}\left( \pm \kappa,(1+a c / c), \mp \kappa c^{-3 / 2}\right)$ besides $E_{0}$, where $\kappa=(1-a b-b / c)^{1 / 2}$.

In the following text, it always assumes that $c(1-a b)-$ $b>0$ is satisfied and only considers the stability of $E_{+}^{*}$ and the other equilibria can be analyzed similarly.

Let $u_{1}=\mathbf{x}-\kappa, u_{2}=\mathbf{y}-(1+a c / c)$, and $u_{3}=\mathbf{z}+\kappa c^{-3 / 2}$, then system (2) becomes

$$
\left\{\begin{array}{l}
\dot{u}_{1}(t)=\left(\frac{1}{c}+k_{1}+k_{2}\right) u_{1}(t)+\kappa u_{2}(t)+u_{3}(t)-k_{1} u_{1}\left(t-\tau_{1}\right) \\
-k_{2} u_{1}\left(t-\tau_{2}\right)+u_{1}(t) u_{2}(t) \\
\dot{u}_{2}(t)=-2 \kappa u_{1}(t)-b u_{2}(t)-u_{1}^{2}(t) \\
\dot{u}_{3}(t)=-u_{1}(t)-c u_{3}(t)
\end{array}\right.
$$

whose characteristic equation is

$$
\begin{aligned}
\nabla\left(\lambda, \tau_{1}, \tau_{2}\right)= & \lambda^{3}+a_{2} \lambda^{2}+a_{1} \lambda+a_{0}+k_{1} e^{-\lambda \tau_{1}}\left(\lambda^{2}+b_{1} \lambda+b_{0}\right) \\
& +k_{2} e^{-\lambda \tau_{2}}\left(\lambda^{2}+b_{1} \lambda+b_{0}\right)=0
\end{aligned}
$$

where

$$
\begin{aligned}
& a_{0}=c\left[2 \kappa^{2}-b\left(k_{1}+k_{2}\right)\right] \\
& a_{1}=2 \kappa^{2}+b c-\frac{b}{c}-(b+c)\left(k_{1}+k_{2}\right) \\
& a_{2}=b+c-\left(\frac{1}{c}+k_{1}+k_{2}\right) \\
& b_{0}=b c \\
& b_{1}=b+c
\end{aligned}
$$

Now, we use the method in $[49,50]$ to study the root distribution of (5). When $\tau_{1}=\tau_{2}=0$, (5) becomes

$$
\begin{aligned}
\nabla(\lambda, 0,0)= & \lambda^{3}+\left(k_{1}+k_{2}+a_{2}\right) \lambda^{2}+\left(k_{1} b_{1}+k_{2} b_{1}+a_{1}\right) \lambda \\
& +k_{1} b_{0}+k_{2} b_{0}+a_{0}=0 .
\end{aligned}
$$

By Routh-Hurwitz criterion, all roots of (7) have negative real parts if and only if

$$
\begin{aligned}
(H 1) a_{2}+k_{1}+k_{2} & >0 \\
a_{0}+k_{1} b_{0}+k_{2} b_{0} & >0 \\
\left(a_{2}+k_{1}+k_{2}\right)\left(a_{1}+k_{1} b_{1}+k_{2} b_{1}\right) & >a_{0}+k_{1} b_{0}+k_{2} b_{0},
\end{aligned}
$$

holds.

2.1. The Case $\tau_{1}>0$ and $\tau_{2}=0$. In this part, let $\tau_{2}=0$, and choose $\tau_{1}$ as the parameter to study the distribution of the 

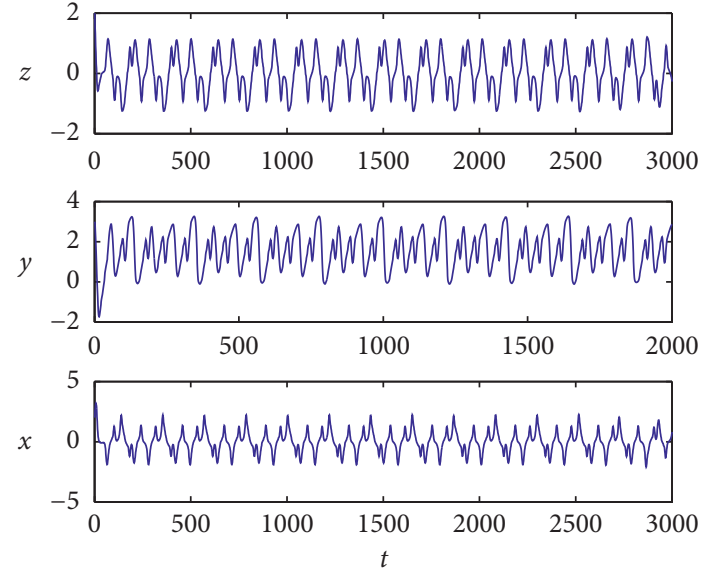

(a)

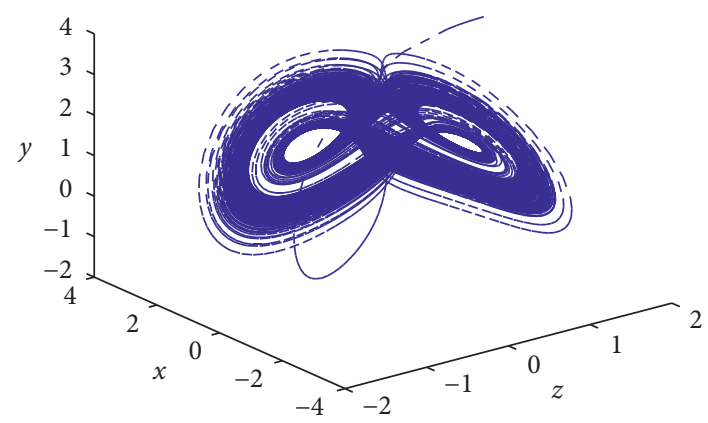

(b)

Figure 1: There exists chaotic attractor for system (1). (a) Time series of the solutions of system (1). (b) Three-dimensional phase diagram of system (1).

root of (5). Let $\mathbf{i} \omega$ be the root of (5), then $\omega$ must satisfy the following equations:

$$
\left\{\begin{array}{l}
-\omega^{3}+\left(a_{1}+k_{2} b_{1}\right) \omega=k_{1}\left[\left(b_{0}-\omega^{2}\right) \sin \omega \tau_{1}-b_{1} \omega \cos \omega \tau_{1}\right] \\
a_{2} \omega^{2}-a_{0}-k_{2}\left(b_{0}-\omega^{2}\right)=k_{1}\left[\left(b_{0}-\omega^{2}\right) \cos \omega \tau_{1}+b_{1} \omega \sin \omega \tau_{1}\right] .
\end{array}\right.
$$

Adding the squares of both sides of (9), it yields to

$$
\omega^{6}+p \omega^{4}+q \omega^{2}+r=0
$$

where

$$
\begin{aligned}
& p=\left(k_{2}+a_{2}\right)^{2}-2\left(a_{1}+k_{2} b_{1}\right)-k_{1}^{2}, \\
& q=\left(a_{1}+k_{2} b_{1}\right)^{2}-2\left(a_{2}+k_{2}\right)\left(a_{0}+b_{0} k_{2}\right)+2 b_{0} k_{1}-k_{1}^{2} b_{1}^{2}, \\
& \boldsymbol{r}=\left(a_{0}+k_{2} b_{0}\right)^{2}-k_{1}^{2} b_{0}^{2} .
\end{aligned}
$$

Furthermore, from (9), it can be obtained that

$$
\left\{\begin{array}{l}
\cos \omega \tau_{1}=\frac{\mathbb{Q}\left(b_{0}-\omega^{2}\right)-p b_{1}}{k_{1}\left(b_{0}-\omega^{2}\right)^{2}+k_{1} b_{1}^{2} \omega^{2}}:=\mathbb{S}_{1}, \\
\sin \omega \tau_{1}=\frac{p\left(b_{0}-\omega^{2}\right)+Q b_{1} \omega}{k_{1}\left(b_{0}-\omega^{2}\right)^{2}+k_{1} b_{1}^{2} \omega^{2}}:=\mathbb{S}_{2},
\end{array}\right.
$$

where

$$
p=-\omega^{3}+\left(a_{1}+k_{2} b_{1}\right) \omega
$$

Q $=a_{2} \omega^{2}-a_{0}-k_{2}\left(b_{0}-\omega^{2}\right)$.

Let $\hbar=\omega^{2}$, then (10) becomes

$$
\mathbf{h}(\varkappa):=\varkappa^{3}+p \varkappa^{2}+q \varkappa+r=0 .
$$

Applying the results in [49], the following conclusions hold.

\section{Lemma 2.}

(i) If $\mu \geq 0$ and $\triangle \leq 0$ hold, then (13) has no positive root (ii) If $\mu<0$ holds, then (13) has at least a positive root

(iii) If $r \geq 0$ and $\triangle>0$ hold, then (13) has a positive roots iff $\varkappa_{1}^{*}>0$ and $\mathbf{h}\left(\varkappa_{1}^{*}\right) \leq 0$, where $\varkappa_{1}^{*}=(-p+\sqrt{\triangle}) / 3$ and $\triangle=p^{2}-3 q$

Without loss of generality, it supposes that (13) has three positive roots, denoted by $\varkappa_{1}, \varkappa_{2}$, and $\varkappa_{3}$, respectively. Then, (10) has three positive roots $\omega_{k}=\sqrt{\varkappa_{k}}(k=1,2,3)$. Substituting $\omega_{k}$ into (9) gives

$$
\tau_{1 k}^{(j)}= \begin{cases}\frac{1}{\omega_{k}}\left\{\arccos \left(\mathbb{S}_{1}\right)+2 j \pi\right\}, & \mathbb{S}_{2} \geq 0, \\ \frac{1}{\omega_{k}}\left\{-\arccos \left(\mathbb{S}_{1}\right)+2(j+1) \pi\right\}, & \mathbb{S}_{2}<0,\end{cases}
$$

where $k=1,2,3, j=0,1, \ldots$

Define $\tau_{1}^{0}=\min _{k=1,2,3}\left\{\tau_{1 k}^{(0)}\right\}$. Let $\lambda\left(\tau_{1}\right)=\gamma\left(\tau_{1}\right)+\mathbf{i} \omega\left(\tau_{1}\right)$ be the root of (5) with $\tau_{2}=0$ satisfying $\gamma\left(\tau_{1 k}^{(j)}\right)=0$ and $\omega\left(\tau_{1 k}^{(j)}\right)=\omega_{k}$.

Lemma 3. Suppose that $\mathbf{h}^{\prime}\left(\varkappa_{k}\right) \neq 0$, then $\left(\mathbf{d}\left(\gamma\left(\tau_{1 k}^{(j)}\right)\right)\right) / \mathbf{d} \tau_{1}$ $\neq 0$ and $\operatorname{Sign}\left\{\mathbf{d}\left(\gamma\left(\tau_{1 k}^{(j)}\right)\right) / \mathbf{d} \tau_{1}\right\}=\operatorname{Sign}\left\{\mathbf{h}^{\prime}\left(\kappa_{k}\right)\right\}$.

Proof. Let $\tau_{2}=0$, and differentiate both sides of (5) about $\tau_{1}$, and it has

$$
\begin{aligned}
{\left[\frac{\mathbf{d} \lambda}{\mathbf{d} \tau_{1}}\right]^{-1}=} & \frac{\left[3 \lambda^{2}+2\left(b_{1}+k_{2}\right) \lambda+a_{1}+k_{2} b_{1}\right] e^{\lambda \tau_{1}}}{k_{1} \lambda\left(\lambda^{2}+b_{1} \lambda+b_{0}\right)} \\
& +\frac{2 \lambda+b_{1}}{\lambda\left(\lambda^{2}+b_{1} \lambda+b_{0}\right)}-\frac{\tau_{1}}{\lambda}
\end{aligned}
$$

Hence, 


$$
\begin{aligned}
{\left[\frac{\mathbf{d}\left(\gamma\left(\tau_{1 k}^{(j)}\right)\right)}{\mathbf{d} \tau_{1}}\right]^{-1} } & =\left.\operatorname{Re}\left\{\frac{\left[3 \lambda^{2}+2\left(b_{1}+k_{2}\right) \lambda+a_{1}+k_{2} b_{1}\right] e^{\lambda \tau_{1}}+k_{1}\left(2 \lambda+b_{1}\right)}{k_{1} \lambda\left(\lambda^{2}+b_{1} \lambda+b_{0}\right)}-\frac{\tau_{1}}{\lambda}\right\}\right|_{\tau_{1}=\tau_{1 k}^{(j)}} \\
& =\frac{1}{\Gamma}\left(3 \omega_{k}^{6}+2 p \omega_{k}^{4}+q \omega_{k}^{2}\right)=\frac{z_{k}}{\Gamma} \mathbf{h}^{\prime}\left(\varkappa_{k}\right),
\end{aligned}
$$

where $\Gamma=k_{1}^{2}\left[b_{1}^{2} \omega_{k}^{4}+\left(\omega_{k}^{2}-b_{0}\right) \omega_{k}^{2}\right]$. Since $\Gamma>0$ and $\varkappa_{k}>0$, then we have

$$
\operatorname{Sign}\left\{\frac{\mathbf{d}\left(\gamma\left(\tau_{1 k}^{(j)}\right)\right)}{\mathbf{d} \tau_{1}}\right\}=\operatorname{Sign}\left\{\mathbf{h}^{\prime}\left(\varkappa_{k}\right)\right\} .
$$

By 3 and applying the Hopf bifurcation theorem in [51], for system (2) it has the following theorem.

Theorem 1. It assumes that (H1) holds:

(i) If $r>0$ and $\Delta \leq 0$ hold, then, for all $\tau_{1} \geq 0, E_{+}^{*}$ is locally asymptotically stable (LAS)

(ii) If either $\mu<0$ or $\mu \geq 0$ and $\triangle>0, \varkappa_{1}^{*}>0, \mathbf{h}\left(\varkappa_{1}^{*}\right) \leq 0$ hold, then for $\tau_{1} \in\left[0, \tau_{1}^{0}\right), E_{+}^{*}$ is LAS

(iii) If all conditions in (ii) and $\mathbf{h} /\left(\varkappa_{k}\right) \neq 0$ hold, then system (2) undergoes Hopf bifurcations at $E_{+}^{*}$ when $\tau_{1}=\tau_{1 k}^{(j)}, j=0,1,2, \ldots, k=1,2,3$

We know that the condition (H1) guarantees that all roots of (7) have negative real parts. If (H1) is violated, we define

$$
A=a_{2}+k_{1}+k_{2}, B=a_{1}+\left(k_{1}+k_{2}\right) b_{1}, C=a_{0}+\left(k_{1}+k_{2}\right) b_{0} .
$$

Let $\lambda=\Lambda-A / 3$, then (7) becomes

$$
\Lambda^{3}+p_{1} \Lambda+q_{1}=0
$$

where $p_{1}=B-A^{2} / 3$ and $q_{1}=\left(2 A^{3} / 27\right)-(A B / 3)+C$.

Define

$$
\begin{aligned}
\Delta_{1} & =\left(\frac{p_{1}}{3}\right)^{3}+\left(\frac{q_{1}}{2}\right)^{2}, \\
\alpha & =\sqrt{[3]}-\frac{q_{1}}{2}+\sqrt{\Delta_{1}}, \\
\beta & =\sqrt{[3]}-\frac{q_{1}}{2}-\sqrt{\Delta_{1}} .
\end{aligned}
$$

Then, from Cardano's formula, it has the following Theorem.

\section{Theorem 2.}

(i) If $\triangle_{1}<0$, then (19) has three real roots

(ii) If $\Delta_{1}>0$, then (19) has a real root $\alpha+\beta-A / 3$ and $a$ pair of conjugate complex roots $-(((\alpha+\beta) / 2)+$ $(A / 3)) \pm \mathbf{i}((\sqrt{3}(\alpha-\beta)) / 2)$

Furthermore, we assume that

$$
\begin{aligned}
(H 2) \Delta_{1} & >0, \\
\frac{\alpha+\beta}{2}+\frac{A}{3} & <0, \\
\alpha+\beta-\frac{A}{3} & <0, \\
\alpha-\beta & \neq 0 .
\end{aligned}
$$

Theorem 3. It assumes that (H2) holds. For system (2), it has the following results.

(i) If $\mu>0$ and $\Delta \leq 0$, then, for all $\tau_{1} \geq 0, E_{+}^{*}$ of system (2) is unstable.

(ii) If either $\mu<0$ or $\mu \geq 0$ and $\Delta>0, \varkappa_{1}^{*}>0, \mathbf{h}\left(\varkappa_{1}^{*}\right) \leq 0$ hold, then for $\tau_{1} \in\left[0, \tau_{1}^{0}\right), E_{+}^{*}$ of system (2) is unstable. In addition, if $\mathbf{d R} \operatorname{e} \lambda\left(\tau_{1}^{0}\right) / \mathbf{d} \tau_{1}<0$, then $E_{+}^{*}$ is LAS when $\tau_{1} \in\left(\tau_{1}^{0}, \tau_{1}^{1}\right)$, where $\tau_{1}^{1}$ is the second critical value.

(iii) If all conditions in (ii) and $\mathbf{h} /\left(\varkappa_{k}\right) \neq 0$ hold, then system (2) undergoes Hopf bifurcations at $E_{+}^{*}$ when $\tau_{1}=\tau_{1 k}^{(j)}, j=0,1,2, \ldots, k=1,2,3$.

From the abovementioned discussion, one can know that the stable switch may exist as $\tau_{1}$ varies for system (2) with $\tau_{2}=0$. Define $I$ as stable interval of $\tau_{1}$.

2.2. The Case $\tau_{1} \in I$ and $\tau_{2}>0$. In this part, let $\tau_{1} \in I, \tau_{2}>0$, and $\lambda=\mathbf{i}\left(\omega=\omega\left(\tau_{2}\right)>0\right)$ be the root of (5), and it has

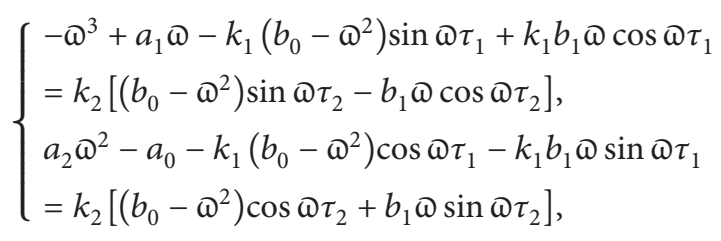

which yields to

$$
\left\{\begin{array}{l}
\cos \omega \tau_{2}=\frac{Q^{1}\left(b_{0}-\Phi^{2}\right)-p^{1} b_{1}}{k_{2}\left(b_{0}-\Phi^{2}\right)^{2}+k_{2} b_{1}^{2} \Phi^{2}}:=\mathbb{T}_{1}, \\
\sin \omega \tau_{2}=\frac{p^{1}\left(b_{0}-\Phi^{2}\right)+Q^{1} b_{1} \Phi}{k_{2}\left(b_{0}-\Phi^{2}\right)^{2}+k_{2} b_{1}^{2} \Phi^{2}}:=\mathbb{T}_{2},
\end{array}\right.
$$

where 


$$
R^{1}=-\varpi^{3}+a_{1} \varpi-k_{1}\left(b_{0}-\varpi^{2}\right) \sin \varpi \tau_{1}+k_{1} b_{1} \varpi \cos \varpi \tau_{1},
$$

and

$$
Q^{1}=a_{2} \Phi^{2}-a_{0}-k_{1}\left(b_{0}-\Phi^{2}\right) \cos \bowtie \tau_{1}-k_{1} b_{1} \bowtie \sin \oplus \tau_{1} .
$$

Hence, we have

$$
\begin{aligned}
& g(\varpi):=\varpi^{6}+\left(b_{1}^{2}-2 a_{1}-k_{2}^{2}+k_{1}^{2}\right) \varpi^{4}+\left[a_{1}^{2}-2 a_{0} b_{1}\right. \\
& \left.+\left(2 b_{0}-b_{1}^{2}\right)\left(k_{2}^{2}-k_{1}^{2}\right)\right] \Phi^{2} \\
& +a_{0}^{2}-b_{0}^{2}\left(k_{2}^{2}-k_{1}^{2}\right)+2\left[k_{1}\left(a_{0}-b_{1} \varpi^{2}\right)\left(b_{0}-\varpi^{2}\right)\right. \\
& \left.+k_{1} b_{1} \bowtie\left(a_{1} \bowtie-\varpi^{3}\right)\right] \cos \bowtie \tau_{1} \\
& +2\left[k_{1} b_{1} \oplus\left(a_{0}-b_{1} \varpi^{2}\right)-k_{1}\left(a_{1} \omega-\varpi^{3}\right)\left(b_{0}-\varpi^{2}\right)\right] \\
& \sin \bowtie \tau_{1}=0 \text {, }
\end{aligned}
$$

with $g(0)=a_{0}^{2}-b_{0}^{2}\left(k_{2}^{2}-k_{1}^{2}\right)+2\left[k_{1}\left(a_{0}-b_{1} \varpi^{2}\right)\left(b_{0}-\omega^{2}\right)+\right.$ $\left.k_{1} b_{1} @\left(a_{1} \bowtie-\Phi^{3}\right)\right]$ and $g(+\infty)=+\infty$.

It can be easily known that equation (26) has at most $N$ positive roots, denoted by $\Phi_{k}(k=1,2, \ldots, N)$. From (22), we have

$$
\tau_{2 k}^{(i)}= \begin{cases}\frac{1}{\varpi_{k}}\left\{\arccos \left(\mathbb{T}_{1}\right)+2 i \pi\right\}, & \mathbb{T}_{2} \geq 0, \\ \frac{1}{\varpi_{k}}\left\{-\arccos \left(\mathbb{T}_{1}\right)+2(i+1) \pi\right\}, & \mathbb{T}_{2}<0, i=0,1,2, \ldots\end{cases}
$$

Denote

$$
\begin{aligned}
\tau_{2}^{0} & =\tau_{2 k_{0}}^{(0)}=\min _{k \in\{1,2, \ldots, N\}}\left\{\tau_{2 k}^{(0)}\right\}, \\
\varpi_{0} & =\varpi_{k_{0}} .
\end{aligned}
$$

Let $\lambda\left(\tau_{2}\right)=\alpha\left(\tau_{2}\right)+\mathbf{i} \oplus\left(\tau_{2}\right)$ be the root of (5) satisfying $\alpha\left(\tau_{2 k}^{(j)}\right)=0$ and $\omega\left(\tau_{2 k}^{(j)}\right)=\varpi_{k}$. By computation, we obtain

$$
\begin{aligned}
& \alpha \prime\left(\tau_{2}^{0}\right)=\sigma\left\{R S-T U-2 k_{2}^{2} \varpi_{0}^{2}+2 b_{0} k_{2}^{2} \varpi_{0}^{2}-b_{1}^{2} k_{2}^{2} \varpi_{0}^{2}+\left(R D \varpi_{0}-E T \varpi_{0}\right) \sin \varpi_{0} \tau_{2}^{0}\right. \\
& -(Q D+P E) \varpi_{0} \cos \varpi_{0}\left(\tau_{1}+\tau_{2}^{0}\right)+(-E Q+P D) \varpi_{0} \\
& \left.\sin \varpi_{0}\left(\tau_{1}+\tau_{2}^{0}\right)-\left(E R \varpi_{0}+D T \varpi_{0}\right) \cos {\varpi_{0}}_{0}^{0}+(S P+U Q) \cos \varpi_{0} \tau_{1}+(Q S-P U) \sin \varpi_{0} \tau_{1}\right\}^{-1} \text {, }
\end{aligned}
$$

where

$$
\begin{aligned}
& \widetilde{\sigma}=b_{1}^{2} k_{2}^{2} \omega_{0}^{4}+\left(b_{0}^{2}-\varpi_{0}^{2}\right)^{2} k_{2}^{2} \varpi_{0}^{2}, \\
& P=-k_{1}\left(-b_{1}+b_{0} \tau_{1}-\tau_{1} \oplus_{0}^{2}\right), \\
& Q=-k_{1}\left(-2 \varpi_{0}+b_{1} \tau_{1} \varpi_{0}\right), \\
& R=-3 \oplus_{0}^{2}+a_{1} \text {, } \\
& D=-k_{1}\left(b_{0}-\varpi_{0}\right) \text {, } \\
& E=-k_{1} b_{1} \omega_{0} \text {, } \\
& S=a_{1} \oplus_{0}^{2}-\omega_{0}^{4}, \\
& T=2 b_{1} \varpi_{0} \text {, } \\
& U=-b_{1} \omega_{0}^{3}+a_{0} \omega_{0} .
\end{aligned}
$$

To sum up, we have the following theorem.

Theorem 4. Suppose that either (H1) or (H2) is satisfied, and $\tau_{1} \in I$ for system (2).

(i) If (26) has no positive roots, then for all $\tau_{2} \geq 0$, $E_{+}^{*}$ is LAS.

(ii) If (26) has positive roots, then $E_{+}^{*}$ of is LAS when $\tau_{2} \in\left[0, \tau_{2}^{0}\right)$. In addition, if $\alpha \prime\left(\tau_{2}^{0}\right) \neq 0$, then system (2) undergoes Hopf bifurcation at $E_{+}^{*}$ when $\tau_{2}=\tau_{2}^{0}$.

Remark 1. Obviously, there exists a Hopf bifurcation at $\tau_{2}^{0}$ when $\tau_{1}$ is fixed in the stable interval $I$. However, if we choose $\tau_{1}$ in the unstable interval, then there may be no $\tau_{2}^{*}$ such that when system (2) is unstable in $\tau_{2} \in\left[0, \tau_{2}^{*}\right)$, it is stable in $\tau_{2}>\tau_{2}^{*}$. The result will be discussed in the latter section by using the stability crossing curve method in [52].

Remark 2. For some $\tau_{1}$ and $\tau_{2}$, if (5) has two pairs of purely imaginary roots $\pm \mathbf{i} w_{1}$ and $\pm \mathbf{i} w_{2}$, all the other roots have negatively real parts. Let $w_{1}: w_{2}=l_{1}: l_{2}$; then, system (2) undergoes a double Hopf bifurcation (DHB) with the ratio $l_{1}: l_{2}$. If $l_{1}, l_{2} \in \mathbb{Z}^{+}$, then it is called a resonant DHB; otherwise, it is called a nonresonant DHB. Since in system (2) there are several parameters besides $\tau_{1}$ and $\tau_{2}$, the co-dimension 2 bifurcation may occur. An interesting study can be found in [53].

\section{Property of Hopf Bifurcation at $E_{+}^{*}$}

In Section 3, we have obtained some sufficient conditions to guarantee that the Hopf bifurcation occurs in system (2) at $E_{+}^{*}$ when $\tau_{2}=\tau_{2}^{0}$. In this section, we assume that Theorem 4 (ii) is satisfied to establish the explicit formula for the property of Hopf bifurcation at $\tau_{2}=\tau_{2}^{0}$ using the method proposed by Hassard et al. [54].

For convenience, we assume $\tau_{1}>\tau_{2}$ and the phase space $C=C\left(\left[-\tau_{1}, 0\right], \mathbb{R}^{3}\right)$. Let $\bar{\tau}_{2}=\tau_{2}^{0}+\vartheta, \vartheta \in \mathbb{R}$ and dropping “-". Then, system (2) occurs Hopf bifurcation at $\mathcal{Y}=0$. System (2) can be transformed into the following system:

$$
\dot{\mathscr{U}}_{t}=\mathbb{L}_{\vartheta}\left(\mathscr{U}_{t}\right)+f\left(\vartheta, \mathscr{U}_{t}\right)
$$

where $\mathscr{U}_{t}(\theta)=\mathscr{U}(t+\theta) \in C$, and $\mathbb{L}_{\vartheta}: C \longrightarrow \mathbb{R}^{3}, f: \mathbb{R} \times$ $C \longrightarrow \mathbb{R}^{3}$ are given, respectively, by 


$$
\mathbb{L}_{9} \varphi=A_{1} \varphi(0)+B_{1} \varphi\left(-\tau_{1}\right)+B_{2} \varphi\left(-\tau_{2}^{0}\right),
$$

where

$$
\begin{aligned}
A_{1} & =\left(\begin{array}{ccc}
k_{1}+k_{2}+\frac{1}{c} & \kappa & 1 \\
-2 \kappa & -b & 0 \\
-1 & 0 & -c
\end{array}\right), \\
B_{1} & =\left(\begin{array}{ccc}
-k_{1} & 0 & 0 \\
0 & 0 & 0 \\
0 & 0 & 0
\end{array}\right), \\
B_{2} & =\left(\begin{array}{ccc}
-k_{2} & 0 & 0 \\
0 & 0 & 0 \\
0 & 0 & 0
\end{array}\right), \\
f(\vartheta, \varphi) & =\left(\begin{array}{c}
\varphi_{1}(0) \varphi_{2}(0) \\
-\varphi_{1}^{2}(0) \\
0
\end{array}\right),
\end{aligned}
$$

where $\varphi=\left(\varphi_{1}, \varphi_{2}, \varphi_{3}\right)^{T} \in C$.

By the Riesz representation theorem, for $\Theta \in\left[-\tau_{1}, 0\right]$, there exists a bounded variation function $\zeta(\Theta, \vartheta)$ such that

$$
\mathbb{L}_{\vartheta} \varphi=\int_{-\tau_{1}}^{0} \mathbf{d} \zeta(\Theta, \vartheta) \varphi(\Theta)
$$

In fact, one may choose

$$
\zeta(\Theta, \vartheta)= \begin{cases}0, & \Theta=-\tau_{1}, \\ B_{1}, & \Theta \in\left(-\tau_{1},-\tau_{2}^{0}\right], \\ B_{1}+B_{2}, & \Theta \in\left(-\tau_{2}^{0}, 0\right), \\ A_{1}+B_{1}+B_{2}, & \Theta=0 .\end{cases}
$$

For $\varphi \in C^{1}\left(\left[-\tau_{1}, 0\right], R^{3}\right)$, define

$$
\begin{aligned}
& \mathbb{A}(\vartheta) \varphi= \begin{cases}\int_{-\tau_{1}}^{0} \mathbf{d} \zeta(s, \vartheta) \varphi(s), & \Theta=0, \\
\dot{\varphi}(\Theta), & \Theta \in\left[-\tau_{1}, 0\right),\end{cases} \\
& \mathbb{R}(\vartheta) \varphi= \begin{cases}f(\vartheta, \varphi), & \Theta=0, \\
0, & \Theta \in\left[-\tau_{1}, 0\right) .\end{cases}
\end{aligned}
$$

For $\mathscr{U}_{t}=\mathscr{U}(t+\theta) \in C^{1}$, it has $\mathbf{d} \mathcal{U}_{t} / \mathbf{d} \Theta=\mathbf{d} \mathcal{U}_{t} / \mathbf{d} t$. Then, system (31) can be rewritten as

$$
\dot{\mathcal{U}}_{t}=\mathbb{A}(\vartheta) \mathcal{U}_{t}+\mathbb{R}(\vartheta) \mathcal{U}_{t}
$$

where $\mathscr{U}_{t}(\theta)=\mathscr{U}(t+\theta)$

For $\alpha_{1} \in C\left(\left[-\tau_{1}, 0\right], \mathbb{R}^{3}\right)$ and $\psi, \alpha_{2} \in C^{1}\left(\left[0, \tau_{1}\right], \mathbb{R}^{3 *}\right)$, define

$$
\mathbb{A}^{*} \psi(s)= \begin{cases}\int_{-\tau_{1}}^{0} \mathbf{d} \zeta^{\mathrm{T}}(t, 0) \psi(-t), & s=0, \\ -\dot{\psi}(\jmath), & s \in\left(0, \tau_{1}\right],\end{cases}
$$

and the inner product

$$
\left\langle\alpha_{1}, \alpha_{2}\right\rangle=\bar{\alpha}_{1}(0) \alpha_{2}(0)-\int_{-\tau_{1}}^{0} \int_{\eta=0}^{\Theta} \bar{\alpha}_{1}(\eta-\Theta) \mathbf{d} \zeta(\Theta) \alpha_{2}(\eta) \mathbf{d} \eta,
$$

where $\zeta(\Theta)=\zeta(\Theta, 0)$. By direct computations, we obtain that $q(\Theta)=(1, v, \varsigma)^{\mathrm{T}} e^{\mathrm{i} \omega_{0} \Theta}$ is an eigenvector of $\mathbb{A}$ corresponding to the eigenvalue $i \omega_{0}$, and $q^{*}(\eta)=\overline{\mathscr{D}}$ $\left(1, \nu^{*}, \varsigma^{*}\right) e^{\mathrm{i} \oplus_{0} \eta}$ is an eigenvector of $\mathbb{A}^{*}$ corresponding to the eigenvalue $-\mathbf{i} \Phi_{0}$. Furthermore, it has that

$$
\begin{aligned}
& \left\langle q^{*}(\eta), q(\Theta)\right\rangle=1, \\
& \left\langle q^{*}(\eta), \bar{q}(\Theta)\right\rangle=0,
\end{aligned}
$$

where

$$
\begin{aligned}
\nu & =-\frac{2 \kappa}{b+\mathbf{i} \omega_{0}}, \\
\varsigma & =-\frac{1}{c+\mathbf{i} \omega_{0}}, \\
v^{*} & =\frac{\kappa}{b-\mathbf{i} \omega_{0}}, \\
\varsigma^{*} & =\frac{1}{c-\mathbf{i} \varpi_{0}}, \\
\mathscr{D} & =\left[1+\nu^{*} \bar{\nu}^{*}+\varsigma^{*} \varsigma^{*}-\tau_{1} k_{1} e^{-\mathbf{i} \omega_{0} \tau_{1}}-\tau_{2}^{0} k_{2} e^{-\mathbf{i} \omega_{0} \tau_{2}^{0}}\right]^{-1} .
\end{aligned}
$$

Let $\mathscr{U}_{t}$ be the solution of system (31) when $\vartheta=0$. Define $\mathscr{Z}(t)=\left\langle q^{*}, \mathscr{U}_{t}\right\rangle$; then,

$$
\dot{\mathscr{Z}}(t)=\mathbf{i} \omega_{0} \mathscr{Z}(t)+\bar{q}^{*}(0) \hat{f}(\mathscr{Z}, \overline{\mathscr{Z}}),
$$

where

$$
\widehat{f}=f(0, \mathbb{W}(\mathscr{Z}, \overline{\mathscr{Z}})+2 \operatorname{Re}\{\mathscr{Z} q\})
$$

$$
\begin{aligned}
& \mathbb{W}(\mathscr{Z}, \overline{\mathscr{Z}})=\mathscr{U}_{t}-2 \operatorname{Re}\{\mathscr{Z} q\}, \\
& \mathbb{W}(\mathscr{Z}, \overline{\mathscr{E}})=\mathbb{W}_{20} \frac{\mathscr{I}^{2}}{2}+\mathbb{W}_{11} \mathscr{\mathscr { Z }}+\mathbb{W}_{02} \frac{\overline{\mathscr{E}}^{2}}{2}+\cdots .
\end{aligned}
$$

Rewriting (42) as

$$
\dot{\mathcal{U}}_{t}=\mathrm{i} \oplus_{0} \mathscr{Z}(t)+\mathscr{Z}(\mathscr{Z}, \overline{\mathscr{Z}}),
$$

where

$$
\mathscr{L}(\mathscr{Z}, \overline{\mathscr{Z}})=\mathscr{g}_{20} \frac{\mathscr{Z}^{2}}{2}+\mathscr{q}_{11} \mathscr{\mathscr { E }} \overline{\mathscr{Z}}+\mathscr{q}_{02} \frac{\overline{\mathscr{E}}^{2}}{2}+\mathscr{q}_{21} \frac{\mathscr{Z}^{2} \overline{\mathscr{Z}}}{2}+\cdots .
$$

Furthermore, 


$$
\dot{W}=\left\{\begin{array}{ll}
\mathbb{A} W-2 \operatorname{Re}\left\{\bar{q}^{*}(0) \hat{f} q(\Theta)\right\}, & \Theta \in[-\tau, 0), \\
\mathbb{A} W-2 \operatorname{Re}\left\{\bar{q}^{*}(0) \hat{f} q(\Theta)\right\}+\widehat{f}, & \Theta=0,
\end{array} \stackrel{=A W+\mathbb{W}(\mathscr{Z}, \overline{\mathscr{Z}}, \Theta),}{=}\right.
$$

where

$$
\mathbb{M}(\mathscr{Z}, \overline{\mathscr{E}}, \Theta)=\mathbb{U}_{20}(\Theta) \frac{\mathscr{Z}^{2}}{2}+\mathbb{U}_{11}(\Theta) \mathscr{\mathscr { E }} \overline{\mathscr{E}}+\mathbb{M}_{02}(\Theta) \frac{\overline{\mathscr{X}}^{2}}{2}+\cdots
$$

Notice that

$$
\begin{aligned}
& \mathcal{U}_{1}(t)=\mathscr{Z}+\overline{\mathscr{E}}+\mathbb{W}_{20}^{(1)}(0) \frac{\mathscr{Z}^{2}}{2}+\mathbb{W}_{11}^{(1)}(0) \mathscr{Z} \overline{\mathscr{E}}+\cdots, \\
& \mathcal{U}_{2}(t)=\nu \mathscr{Z}+\bar{\nu} \overline{\mathscr{E}}+\mathbb{W}_{20}^{(2)}(0) \frac{\mathscr{Z}^{2}}{2}+\mathbb{W}_{11}^{(2)}(0) \mathscr{Z} \overline{\mathscr{X}}+\cdots .
\end{aligned}
$$

Hence, we can obtain the following important quantities:

$$
\begin{aligned}
\mathscr{L}_{20}= & 2 \mathscr{D}\left(\nu-\bar{\nu}^{*}\right), \mathscr{g}_{11}=\mathscr{D}\left(\nu+\bar{\nu}-2 \bar{v}^{*}\right), \mathscr{g}_{02}=2 \mathscr{D}\left(\bar{\nu}-\bar{\nu}^{*}\right), \\
\mathscr{I}_{21}= & 2 \mathscr{D}\left[\mathbb{W}_{11}^{(2)}(0)+\frac{1}{2} \mathbb{W}_{20}^{(2)}(0)+\mathbb{W}_{20}^{(1)}(0)\left(\left(\frac{1}{2} \bar{\nu}-\bar{\nu}^{*}\right)\right.\right. \\
& \left.+\mathbb{W}_{11}^{(1)}(0)\left(\nu-2 \bar{v}^{*}\right)\right],
\end{aligned}
$$

where

$$
\begin{aligned}
& \mathbb{W}_{20}(\Theta)=\frac{\mathbf{i} q_{20}}{\varpi_{0}} q(0) e^{\mathbf{i} \omega_{0} \Theta}+\frac{\mathbf{i} \bar{g}_{02}}{3 \varpi_{0}} \bar{q}(0) e^{-\mathbf{i} \omega_{0} \Theta}+\mathbb{E}_{1} e^{2 \mathbf{i} \omega_{0} \Theta}, \\
& \mathbb{W}_{11}(\Theta)=-\frac{\mathbf{i} q_{11}}{\varpi_{0}} q(0) e^{\mathrm{i} \varpi_{0} \Theta}+\frac{\mathbf{i} \bar{g}_{11}}{\varpi_{0}} \bar{q}(0) e^{-\mathrm{i} \varpi_{0} \Theta}+\mathbb{E}_{2}, \\
& \mathbb{E}_{1}=\left(\begin{array}{ccc}
\mathbf{G} & -\kappa & -1 \\
2 \kappa & 2 \mathbf{i} \Phi_{0}+b & 0 \\
1 & 0 & 2 \mathbf{i} \Phi_{0}+c
\end{array}\right)^{-1} \times\left(\begin{array}{c}
\nu \\
-1 \\
0
\end{array}\right), \\
& \mathbb{E}_{2}=\left(\begin{array}{ccc}
\frac{1}{c} & \kappa & 1 \\
-2 \kappa & -b & 0 \\
-1 & 0 & -c
\end{array}\right)^{-1} \times\left(\begin{array}{c}
-(\nu+\bar{\nu}) \\
2 \\
0
\end{array}\right),
\end{aligned}
$$

where $\mathbf{G}=2 \mathbf{i} \Phi_{0}-\left(k_{1}+k_{2}+1 / c\right)+k_{1} e^{-2 i \Phi_{0} \tau_{1}}+k_{2} e^{-2 \mathbf{i} \omega \tau_{2}^{0}}$.
Substituting $\mathbb{E}_{1}$ and $\mathbb{E}_{2}$ into $\mathbb{W}_{20}(\Theta)$ and $\mathbb{W}_{11}(\Theta)$, respectively, furthermore, $\mathscr{q}_{21}$ can be computed. Thus, it can obtain the following quantities:

$$
\begin{aligned}
\mathscr{C}_{1}(0) & =\frac{\mathbf{i}}{2 \Phi_{0}}\left(q_{20} q_{11}-2\left|g_{11}\right|^{2}-\frac{\left|g_{02}\right|^{2}}{3}\right)+\frac{q_{21}}{2}, \\
\mathscr{C}_{2} & =-\frac{\operatorname{Re}\left\{\mathscr{C}_{1}(0)\right\}}{\operatorname{Re} \lambda^{\prime}\left(\tau_{2}^{0}\right)}, \\
\mathscr{T}_{2} & =-\frac{\operatorname{Im}\left\{\mathscr{C}_{1}(0)\right\}+\mathscr{C}_{2} \operatorname{Im} \lambda^{\prime}\left(\tau_{2}^{0}\right)}{\varpi_{0}}, \\
\mathscr{B}_{2} & =2 \operatorname{Re}\left\{\mathscr{C}_{1}(0)\right\} .
\end{aligned}
$$

Hence, we have the following result.

Theorem 5. Hopf bifurcation is supercritical (subcritical) if $\mathscr{E}_{2}>0(<0)$. The bifurcation periodic solutions are orbitally stable (unstable) if $\mathscr{B}_{2}<0(>0)$. The period increase (decrease) if $\mathscr{T}_{2}>0(<0)$.

\section{Crossing Curve Method}

The results in Theorem 4 clearly show that the stability of system (2) changes depending on the parameters of system. However, the $\left(\tau_{1}, \tau_{2}\right)$ plane analysis results for bifurcation generation are not obtained by this method in Section 2. Gu et al. [52] gave an effective approach to separate the stable and unstable regions in the $\left(\tau_{1}, \tau_{2}\right)$ plane by using the stability crossing curves. In this part, we carry out the method. on the basis of equation (5), and it can define the following polynomials about $\lambda$ :

$$
\left\{\begin{array}{l}
R_{0}(\lambda)=\lambda^{3}+a_{2} \lambda^{2}+a_{1} \lambda+a_{0}, \\
R_{1}(\lambda)=k_{1}\left(\lambda^{2}+b_{1} \lambda+b_{0}\right), \\
R_{2}(\lambda)=\frac{k_{2}}{k_{1}} R_{1}(\lambda),
\end{array}\right.
$$

satisfying 
(i) $\operatorname{deg}\left(\mathfrak{p}_{0}(\lambda)\right) \geq \max \left\{\operatorname{deg}\left(\mathfrak{p}_{1}(\lambda)\right), \operatorname{deg}\left(\mathfrak{p}_{2}(\lambda)\right)\right\}$

(ii) $p_{0}(0)+p_{1}(0)+p_{2}(0) \neq 0$

(iii) The polynomials $p_{0}(\lambda)$,

$p_{1}(\lambda)$ and $p_{2}(\lambda)$ do not have any common zeros

(iv) $\lim _{\lambda \rightarrow \infty}\left(\left|\frac{p_{1}(\lambda)}{p_{0}(\lambda)}\right|+\left|\frac{p_{2}(\lambda)}{p_{0}(\lambda)}\right|\right)<1$

The following discussions will follow the continuity of the zeros with respect to the delay parameters as stated in the following lemma [52].

Lemma 4. As the delays $\left(\tau_{1}, \tau_{2}\right)$ continuously vary within $\mathbb{R}_{+}^{2}$, the number of zeros (counting multiplicity) of $\Delta\left(\lambda, \tau_{1}, \tau_{2}\right)$ on $\mathbb{C}_{+}$can change only if a zero appears on or across the imaginary axis.

The characteristic equation (5) has the same zeros with the zeros of

$$
\Delta\left(\lambda, \tau_{1}, \tau_{2}\right)=1+\delta_{1}(\lambda) e^{-\lambda \tau_{1}}+\delta_{2}(\lambda) e^{-\lambda \tau_{2}}=0,
$$

where $\delta_{s}(\lambda)=p_{s}(\lambda) / p_{0}(\lambda), s=1,2$. Therefore, in general, we may obtain all the crossing points and directions of crossing from the solutions of $\Delta\left(\lambda, \tau_{1}, \tau_{2}\right)=0$ instead of $\nabla\left(\lambda, \tau_{1}, \tau_{2}\right)=0$. Now, based on the procedure proposed by [52], the procedure is comprised of the following steps.

The first step is to determine the crossing set $\Omega$ of $\omega$ that satisfies the feasibility condition so that the purely imaginary root exists, and geometrically, the vectors that satisfy (54) form a triangle (see Figure 2).

From Figure 2, the crossing set $\Omega$ can be represented as

$$
\begin{aligned}
& \mathbf{L}_{1}(\omega)=\left|\delta_{1}(\mathbf{i} \omega)\right|+\left|\delta_{2}(\mathbf{i} \omega)\right| \geq 1, \\
& \mathbf{L}_{2}(\omega)=\left|\delta_{1}(\mathbf{i} \omega)\right|-\left|\delta_{2}(\mathbf{i} \omega)\right| \leq 1
\end{aligned}
$$

The second step is to determine the inner angles $\theta_{1}, \theta_{2} \in[0, \pi]$ of the triangle in Figure 2. From the cosine law, it has

$$
\left\{\begin{array}{l}
\cos \theta_{1}=\frac{1+\left|\delta_{1}(\mathbf{i} \omega)\right|^{2}-\left|\delta_{2}(\mathbf{i} \omega)\right|^{2}}{2\left|\delta_{1}(\mathbf{i} \omega)\right|} \\
\cos \theta_{2}=\frac{1+\left|\delta_{2}(\mathbf{i} \omega)\right|^{2}-\left|\delta_{1}(\mathbf{i} \omega)\right|^{2}}{2\left|\delta_{2}(\mathbf{i} \omega)\right|}
\end{array}\right.
$$

For any $\omega \in \Omega$, one can obtain $\left(\tau_{1}, \tau_{2}\right)$ from (54) as follows:

$$
\begin{array}{r}
\tau_{1}^{u^{ \pm}}(\omega)=\frac{1}{\omega}\left[\arg \left(\delta_{1}(\mathbf{i} \omega)\right) \pm \theta_{1}+(2 u-1) \pi\right] \geq 0, \\
u=u_{0}^{ \pm}, u_{0}^{ \pm}+1, u_{0}^{ \pm}+2, \ldots,
\end{array}
$$

and

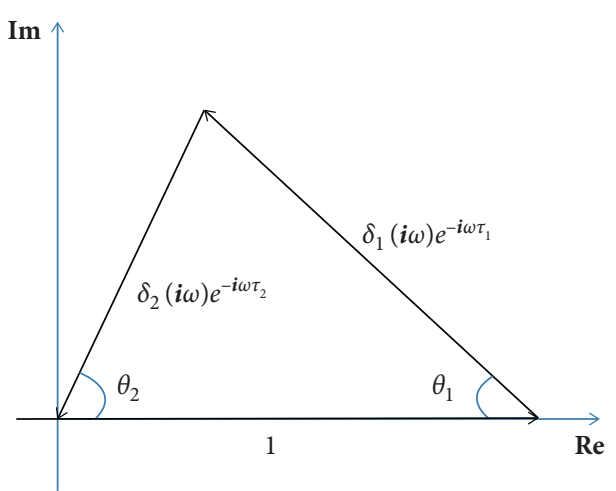

Figure 2: Triangle formed by $1,\left|\delta_{1}(\mathbf{i} \omega)\right|$, and $\left|\delta_{2}(\mathbf{i} \omega)\right|$.

$$
\begin{gathered}
\tau_{2}^{\ell^{ \pm}}(\omega)=\frac{1}{\omega}\left[\arg \left(\delta_{2}(\mathbf{i} \omega)\right) \mp \theta_{2}+(2 \mathscr{b}-1) \pi\right] \geq 0, \\
b=b_{0}^{ \pm}, b_{0}^{ \pm}+1, b_{0}^{ \pm}+2, \ldots,
\end{gathered}
$$

where $u_{0}^{ \pm}$and $\mathscr{b}_{0}^{ \pm}$are the smallest integers so that the right sides of (58) and (59) are nonnegative.

Let

$$
\begin{aligned}
\mathcal{S}_{u, b}^{ \pm}= & \left\{\left(\tau_{1}^{u^{ \pm}}(\omega), \tau_{2}^{\ell^{ \pm}}(\omega)\right)\right\} \\
= & \left\{\left(\frac{1}{\omega}\left[\arg \left(\delta_{1}(\mathbf{i} \omega)\right)+(2 u-1) \pi \pm \theta_{1}\right],\right.\right. \\
& \left.\left.\frac{1}{\omega}\left[\arg \left(\delta_{2}(\mathbf{i} \omega)\right)+(2 \ell-1) \pi \mp \theta_{2}\right]\right)\right\},
\end{aligned}
$$

then

$$
\mathscr{T}_{\omega}=\left(\cup_{u \geq u_{0}^{+}, b \geq b_{0}^{+}} \mathcal{S}_{u, b}^{+}\right) \cup\left(\underset{u \geq u_{0}^{-}, b \geq b_{0}^{-}}{\cup} \mathcal{S}_{u, b}^{-}\right),
$$

which is the set of all $\left(\tau_{1}, \tau_{2}\right)$ such that $\Delta\left(\lambda, \tau_{1} \tau_{2}\right)$ has a zero at $\lambda=\mathbf{i} \omega$.

$\mathscr{T}=\left\{\mathscr{T}_{\omega}: \omega \in \Omega\right\}$ identifies the stability crossing curves in $\left(\tau_{1}, \tau_{2}\right)$ plane, and the crossing set $\Omega$ is composed by a finite number of intervals with finite length. Let these intervals be $\Omega_{k}, k=1,2, \ldots, \mathbf{N}$, arranged in such an order that the left endpoint of $\Omega_{k}$ increases with increasing $k$. Then, $\Omega=\cup_{k=1}^{N} \Omega_{k}$, and the left endpoints of the intervals $\omega_{k}^{l}$ and the right endpoints $\omega_{k}^{r}$ must only satisfy one of the three equations: $L_{1}(\omega)=1$ and $L_{2}(\omega)= \pm 1$.

Let

$$
\begin{aligned}
\mathscr{T}_{u, \ell, k}^{ \pm} & =\bigcup_{\omega \in \Omega_{k}} \mathcal{S}_{u, \ell}^{ \pm}, \\
\mathscr{T}^{k} & =\bigcup_{u=-\infty}^{+\infty} \bigcup_{b=-\infty}^{+\infty}\left(\mathscr{T}_{u, \ell, k}^{+} \cup \mathscr{T}_{u, \ell, k}^{-}\right) \cap \mathbf{R}_{+}^{2} .
\end{aligned}
$$

Then, $\mathscr{T}=\cup_{k=1}^{N} \mathscr{T}^{k}$.

Hence, we can divide these endpoints into three types according to the conditions satisfied by the equation $\omega_{k}^{l}$ or $\omega_{k}^{r}$. If $\omega_{1}^{l}=0$, then $\Omega_{1}$ may have a special type. As stated by [52], the possible shapes of $\mathscr{T}^{k}$ must belong to one of the following three types: 
(i) A series of closed curves.

(ii) A series of spiral-like curves oriented along horizontally, vertically, or diagonally.

(iii) A series of open-ended curves whose ends approach $\infty$.

If the left endpoint of $\Omega_{k}$ is of Type $l$ and its right endpoint is of Type $r$, we call an interval $\Omega_{k}$ is of Type $l r$. There are a total of 12 possible types, where

Type $1: \mathbf{L}_{2}(\omega)=1$ is satisfied. $\mathcal{S}_{u, b, k}^{+}$links $\mathcal{S}_{u, b-1, k}^{-}$at the end.

Type 2: $\mathbf{L}_{2}(\omega)=-1$ is satisfied. $\mathcal{S}_{u, \ell, k}^{+}$links $\mathcal{S}_{u+1, \ell, k}^{-}$at the end.

Type 3: $\mathbf{L}_{1}(\omega)=1$ is satisfied. $\mathcal{S}_{u, \ell, k}^{+}$links $\mathcal{S}_{u, \ell, k}^{-}$at the end.

Type 0: $\omega_{k}^{l}=0$. As $\omega \longrightarrow 0, \mathcal{S}_{u, \ell, k}^{+}$and $\mathcal{S}_{u, \ell, k}^{-}$approach $\infty$.

In 12 possible types, Type 11, Type 22, and Type 33 form a series of closed curves. Type 12 and Type 21, Type 13 and Type 31, and Type 23, and Type 32 form series spiral-like curves oriented along diagonally, vertically, and horizontally, respectively. Type 01, Type 02, and Type 03 form a series of open-ended curves.

Next, to determine the existence of Hopf bifurcation, we consider the direction of the root of (5) through the imaginary axis by the method given in [52]. By (54) and the implicit function theorem, $\tau_{1}$ and $\tau_{2}$ can be expressed as the function of $\lambda=\mathbf{i} \omega$. As $\lambda$ moves along the imaginary axis, $\left(\tau_{1}, \tau_{2}\right)=\left(\tau_{1}^{u^{ \pm}}(\omega), \tau_{2}^{\ell^{ \pm}}(\omega)\right)$ moves along $\mathscr{T}^{k}$. For a fixed $\omega \in \Omega_{k}$, let

$$
\begin{gathered}
\left.\operatorname{Re}\left(\frac{\mathbf{i}}{\lambda} \frac{\partial \Delta\left(\lambda, \tau_{1}, \tau_{2}\right)}{\partial \lambda}\right)\right|_{\lambda=\mathbf{i} \omega}=\mathfrak{R}_{0}, \\
\left.\operatorname{Im}\left(\frac{\mathbf{i}}{\lambda} \frac{\partial \Delta\left(\lambda, \tau_{1}, \tau_{2}\right)}{\partial \lambda}\right)\right|_{\lambda=\mathbf{i} \omega}=\mathfrak{\Im}_{0}, \\
-\left.\operatorname{Re}\left(\frac{1}{\lambda} \frac{\partial \Delta\left(\lambda, \tau_{1}, \tau_{2}\right)}{\partial \tau_{s}}\right)\right|_{\lambda=\mathbf{i} \omega}=\mathfrak{R}_{s}, \\
-\left.\operatorname{Im}\left(\frac{1}{\lambda} \frac{\partial \Delta\left(\lambda, \tau_{1}, \tau_{2}\right)}{\partial \tau_{s}}\right)\right|_{\lambda=\mathbf{i} \omega}=\mathfrak{J}_{s},
\end{gathered}
$$

where $s=1,2$.

The direction in which the $\omega$ increases is called the positive direction of the curve, which is reversed when the curve passes the point corresponding to the $\Omega_{k}$ endpoint. When we move in the positive direction of the curve, we also call the region on the left-hand side the region on the left. The following results come from [52].

Lemma 5. Let $\omega \in\left(\omega_{k}^{l}, \omega_{k}^{r}\right)$ and $\left(\tau_{1}, \tau_{2}\right) \in \mathscr{T}^{k}$ so that $\mathbf{i} \omega$ is a simple root of (5) and for any $\omega \prime \neq \omega, \Delta\left(\mathbf{i} \omega \prime, \tau_{1}, \tau_{2}\right) \neq 0$. Then, as $\left(\tau_{1}, \tau_{2}\right)$ moves from the right-side region to the left-side region of the corresponding curve in $\mathscr{T}^{k}$, a pair of roots of (54) cross the imaginary axis to the right side if $\Re_{2} \mathfrak{I}_{1}-\mathfrak{R}_{1} \mathfrak{\Im}_{2}>0$. If the inequality is reversed, the crossing direction is opposite.
Theorem 6. Let $\omega, \tau_{1}$, and $\tau_{2}$ satisfy the conditions in Lemma 5. Then, when $\left(\tau_{1}, \tau_{2}\right)$ crosses the curve along the direction $\left(\ell_{1}, \ell_{2}\right)$, a pair of roots of (54) cross the imaginary axis to the right side if

$$
\ell_{1}\left(\mathfrak{R}_{0} \mathfrak{\Im}_{1}-\mathfrak{R}_{1} \mathfrak{\Im}_{0}\right)+\ell_{2}\left(\mathfrak{R}_{0} \mathfrak{\Im}_{2}-\mathfrak{R}_{2} \mathfrak{\Im}_{0}\right)>0 .
$$

If the inequality is reversed, the crossing direction is opposite.

\section{Numerical Simulations}

In this part, we will carry out some numerical simulations by using Matlab Microsoft to confirm the theoretical analyses.

Firstly, as an example, we investigate the following system:

$$
\left\{\begin{array}{l}
\dot{x}(t)=(\mathbf{y}-0.9) \mathbf{x}+\mathbf{z}-\left[\mathbf{x}(t)-\mathbf{x}\left(t-\tau_{1}\right)\right]-2\left[\mathbf{x}(t)-\mathbf{x}\left(t-\tau_{2}\right)\right] \\
\dot{y}(t)=1-0.2 \mathbf{y}-\mathbf{x}^{2} \\
\dot{z}(t)=-\mathbf{x}-1.2 \mathbf{z}
\end{array}\right.
$$

and the initial functions are $\varphi_{1}(\theta) \equiv 2, \varphi_{2}(\theta) \equiv 3$, and $\varphi_{3}(\theta) \equiv 2$. With these parameters, condition $(H 2)$ holds. When $\tau_{2}=0$, (10) has two positive roots $\omega_{1} \doteq 0.9752$ and $\omega_{2} \doteq 1.9997$. Substituting them into (14) gives, respectively,

$$
\begin{aligned}
\tau_{11}^{(j)} & =0.3795+6.4430 j, \\
\tau_{12}^{(i)} & =2.5811+3.1421 i, \quad j, i=0,1,2, \ldots
\end{aligned}
$$

Furthermore,

$\mathbf{d}\left(\operatorname{Re} \lambda\left(\tau_{11}^{(j)}\right)\right) / \mathbf{d} \tau_{1}<0$ and $\mathbf{d}\left(\operatorname{Re} \lambda\left(\tau_{12}^{(i)}\right)\right) / \mathbf{d} \tau_{1}>0$. By Theorem $4, E_{+}^{*}$ is unstable when $\tau_{1} \in[0,0.3795) \cup(2.5811,+\infty)$, and LAS when $\tau_{1} \in(0.3795,2.5811)$. The numerical simulation results are shown in Figures 3-5.

Fix $\tau_{1}=2.2 \in(0.3795,2.5811)$, and it computes $\tau_{2}^{0} \doteq 2.4692$. By Theorem 4 , we know that $E_{+}^{*}$ is LAS for $\tau_{2} \in[0,2.4692)$. Choosing $\tau_{2}=1, E_{+}^{*}$ is stable (see Figure 6). Furthermore, by Section 3, it has $\mathscr{C}_{1}(0)=-10.1592+$ $0.7794 \mathbf{i}, \mathscr{B}_{2}<0$ and $\mathscr{E}_{2}>0$ when $\tau_{2}=2.4692$, and the bifurcating periodic solution is stable, which is illustrated in Figure 7.

Next, one gives some examples for the crossing curve method using Matlab Microsoft. Firstly, it still chooses the parameters in system (65). It can obtain the crossing set based on the equation of $\mathbf{L}_{1}(\omega)$ and $\mathbf{L}_{2}(\omega)$. In equations (58) and (59), we regard $\tau_{1}$ and $\tau_{2}$ as the function of $\omega$, by drawing the parametric equation curves in $\left(\tau_{1}, \tau_{2}\right)$ plane, and it obtains the crossing curves. The crossing set has only a interval $\Omega_{1}$ and $\omega_{1}^{l}=0.9893$ and $\omega_{1}^{r}=2.6715$, satisfying $\mathbf{L}_{1}(\omega)=1$ with $\omega=\omega_{1}^{l}$ and $\omega=\omega_{1}^{r}$ (see Figure 8(a)). So, the interval $\Omega_{1}$ is Type 33 and the crossing curves form a series of closed curves (see Figures 8(b) and 8(c)). Firstly, it chooses $\tau_{1}=10>2.5811$ and $\tau_{2}=0$, and it can obtain that $E_{+}^{*}$ is unstable (see Figure 9). In the following, it chooses, respectively, $\tau_{2}=1,3.35,3.7$ for fixed $\tau_{1}=10$, and it can find that $E_{+}^{*}$ is stable when $\tau_{2}=1$ (see Figure 10) and $\tau_{2}=3.7$ (see Figure 11), unstable when $\tau_{2}=3.35$, and there exists a stable periodic solution (see Figure 12). Furthermore, it can fix $\mathrm{a}=0.9, \mathrm{~b}=0.2$, and $\mathrm{c}=1.2$, and let $k_{1}$ and $k_{2}$ change, and 

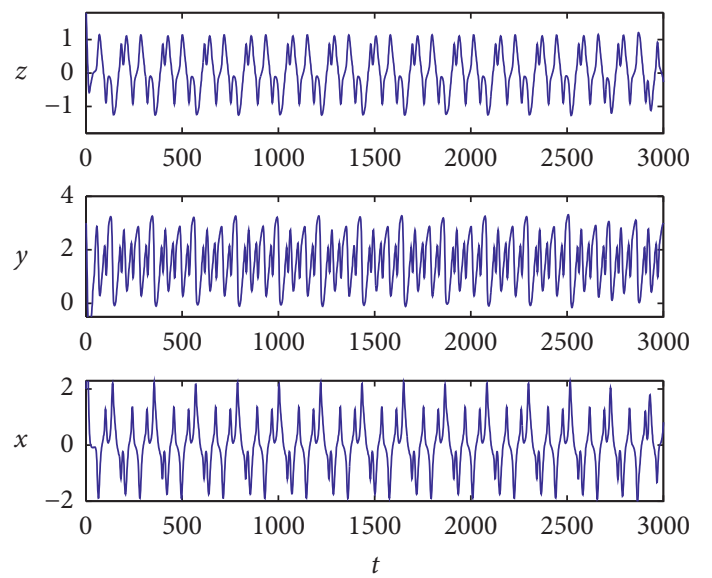

(a)

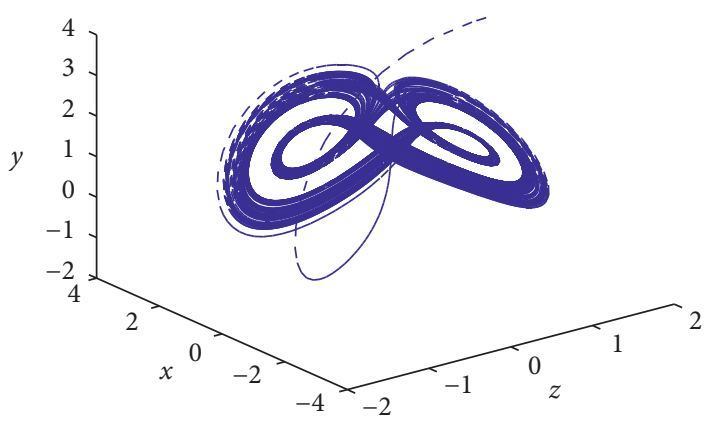

(b)

FIgURE 3: $E_{+}^{*}$ is unstable, and chaos phenomenon still exists for system (65) when $\tau_{1}=0.1 \in[0,0.3795)$. (a) Time series of the solutions. (b) Three-dimensional phase diagram.
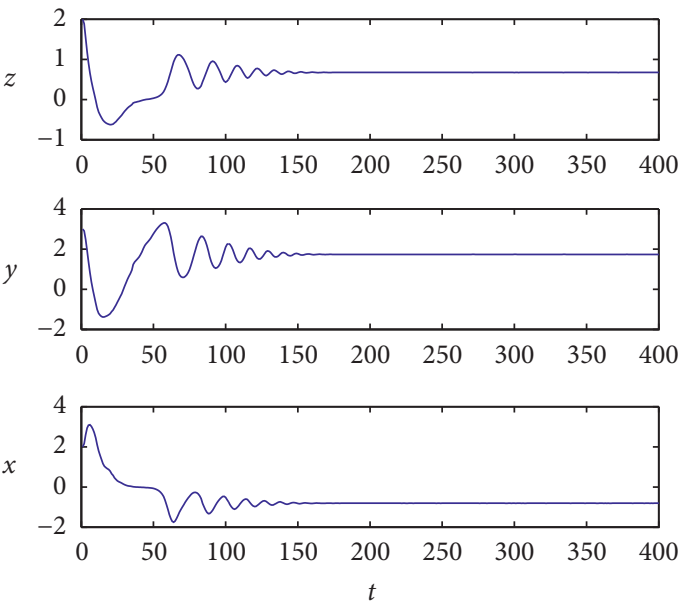

(a)

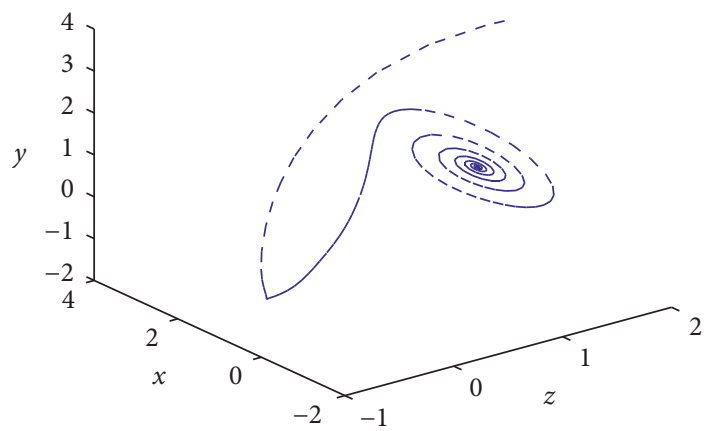

(b)

Figure 4: $E_{+}^{*}$ is stable and the chaos phenomenon disappears for system (65) when $\tau_{1}=0.8 \in(0.3795,2.5811)$. (a) Time series of the solutions. (b) Three-dimensional phase diagram.
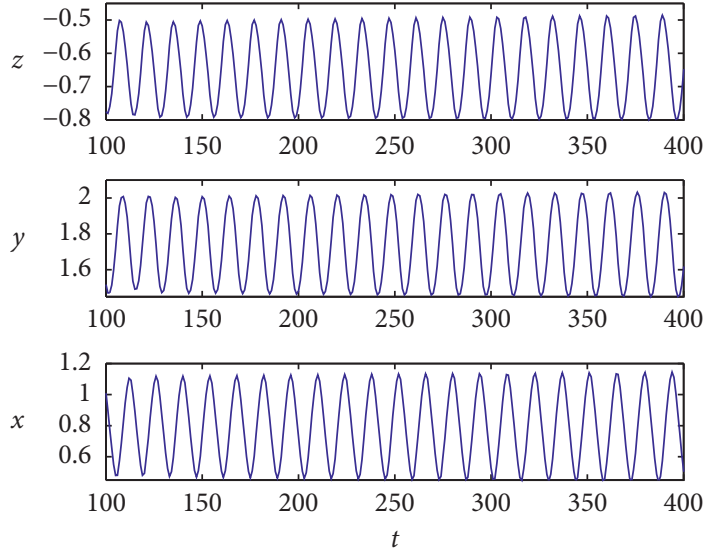

(a)

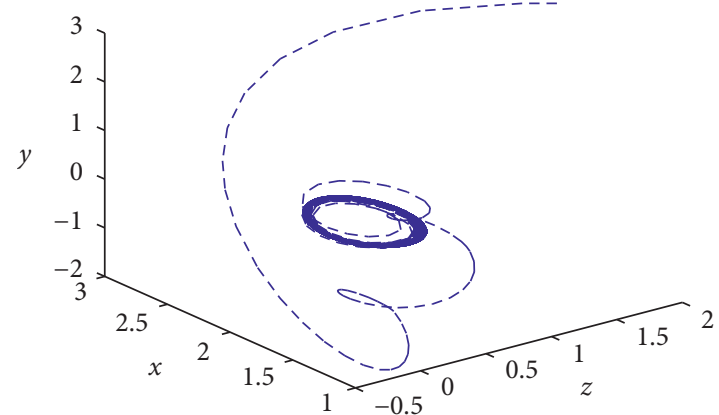

(b)

Figure 5: $E_{+}^{*}$ is unstable and a stable periodic solution exists for system (65) when $\tau_{1}=2.77>2.5811$. (a) Time series of the solutions. (b) Three-dimensional phase diagram. 

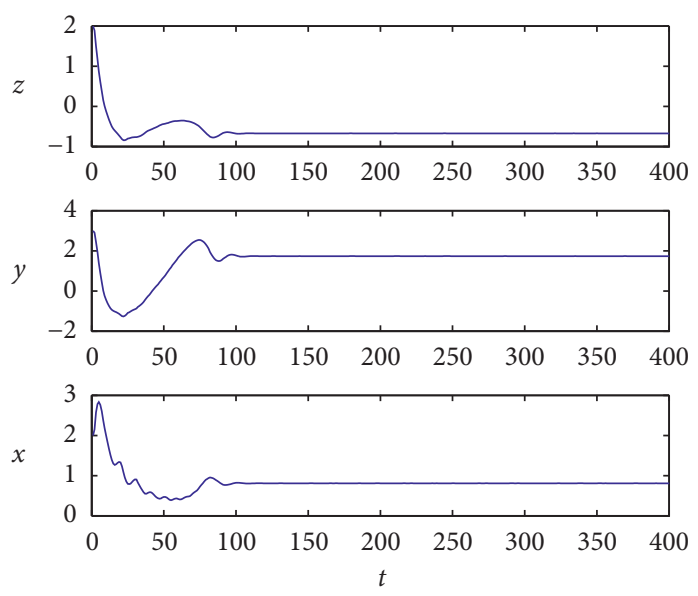

(a)

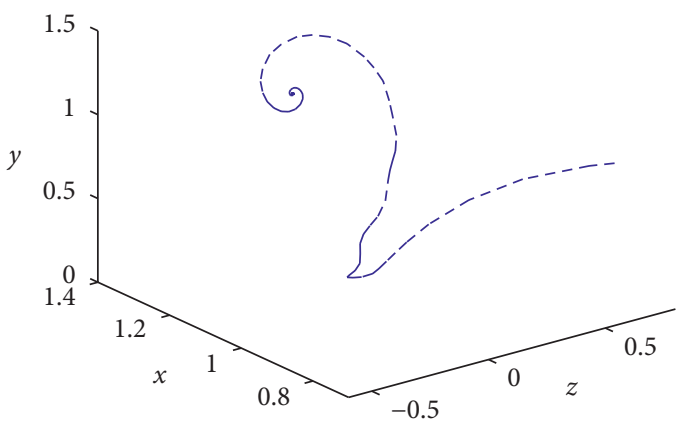

(b)

Figure 6: $E_{+}^{*}$ is stable for system (65) with $\tau_{1}=2.2$ and $\tau_{2}=1$. (a) Time series of the solutions. (b) Three-dimensional phase diagram.
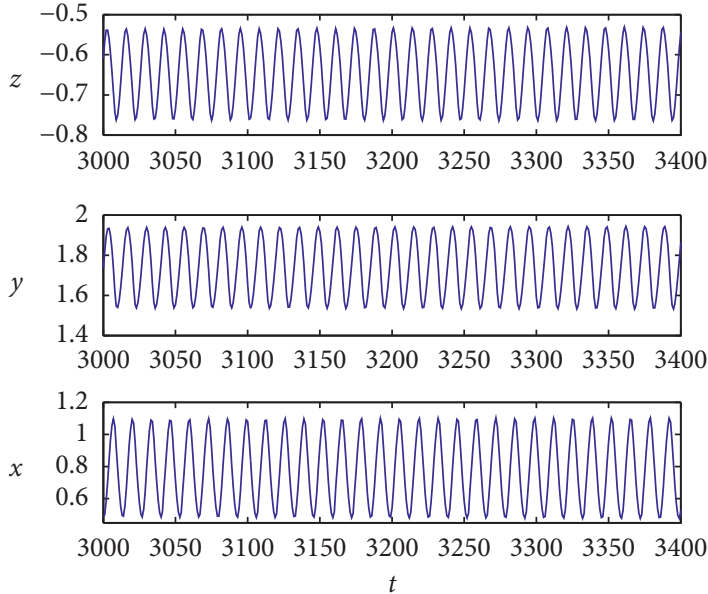

(a)

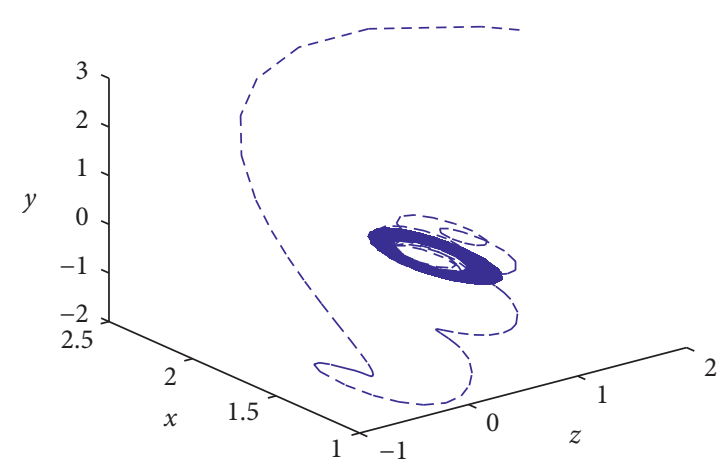

(b)

Figure 7: $E_{+}^{*}$ is unstable and there exists a stable periodic solution for system (65), where $\tau_{1}=2.2$ and $\tau_{2}=\tau_{2}^{0}$. (a) Time series of the solutions. (b) Three-dimensional phase diagram.

the crossing curves can produce different shapes (see Figures 13-15). When $k_{1}=1$ and $k_{2}=-5$, the crossing sets have two intervals $\Omega_{1}=(0.3696,0.9733)$ and $\Omega_{2}=$ $(2.9688,5.4793)$. Here, $\omega_{1}^{r}$ and $\omega_{2}^{l}$ satisfy $\mathbf{L}_{2}(\omega)=-1$ while $\omega_{1}^{l}$ and $\omega_{2}^{r}$ satisfy $\mathbf{L}_{1}(\omega)=1$ (see Figure $13(\mathrm{a})$ ). So, the interval $\Omega_{1}$ belongs to Type 32 and the interval $\Omega_{2}$ belongs to Type 23. The crossing curves are spatial-like curves, as shown in Figures 13(b) and 13(c). Choosing $k_{1}=-4$ and $k_{2}=2$, the crossing sets include two intervals $\Omega_{1}=(0.2097,1.0151)$ and $\Omega_{2}=(2.3341,6.2418)$ (see Figure $14(\mathrm{a})$ ). The crossing curves belongs to Type 31 and Type 13 with the spiral-like shape (see Figure 14(b)). Here, the stability crossing curves in $\Omega_{1}=(0.2097,1.0151)$ belonging to type 31 are not drawn. If $k_{1}=3$ and $k_{2}=1$, the crossing set includes a interval $\Omega_{1}=(0,0.7486)$. Since $\omega_{1}^{l}=0$ and $\mathbf{L}_{1}\left(\omega_{1}^{r}\right)=0$ (see Figure 15(a)), the stability crossing curves belongs to Type 03 with the open-ended shapes (see Figure 15(b)). These show that the changes of the feedback strengths $k_{1}$ and $k_{2}$ can alter the stable region of the system in $\left(\tau_{1}, \tau_{2}\right)$ plane, and it has an important effect on the stability of the financial system.

In addition, for the following two systems:

$$
\left\{\begin{array}{l}
\dot{x}(t)=(\mathbf{y}-a) \mathbf{x}+\mathbf{z} \\
\dot{y}(t)=1-b \mathbf{y}-\mathbf{x}^{2}+k_{1}\left[\mathbf{y}(t)-\mathbf{y}\left(t-\tau_{1}\right)\right] \\
+k_{2}\left[\mathbf{y}(t)-\mathbf{y}\left(t-\tau_{2}\right)\right] \\
\dot{z}(t)=-\mathbf{x}-c \mathbf{z}
\end{array}\right.
$$

and

$$
\left\{\begin{array}{l}
\dot{x}(t)=(\mathbf{y}-a) \mathbf{x}+\mathbf{z}, \\
\dot{y}(t)=1-b \mathbf{y}-\mathbf{x}^{2}, \\
\dot{z}(t)=-\mathbf{x}-c \mathbf{z}+k_{1}\left[\mathbf{z}(t)-\mathbf{z}\left(t-\tau_{1}\right)\right]+k_{2}\left[\mathbf{z}(t)-\mathbf{z}\left(t-\tau_{2}\right)\right],
\end{array}\right.
$$

that is, delayed feedback terms appear on the investment demand or the price index, respectively. Systems (67) and 


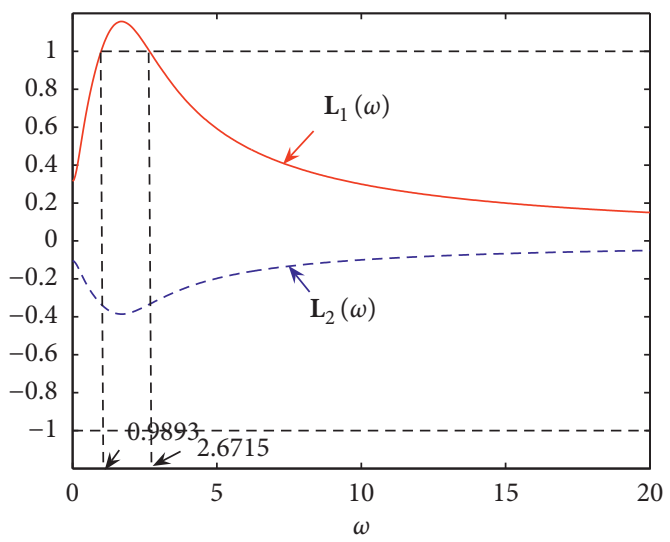

(a)

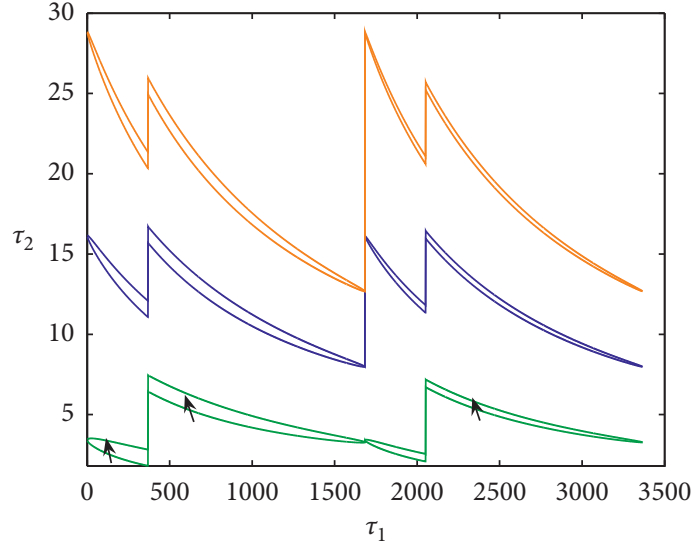

(b)

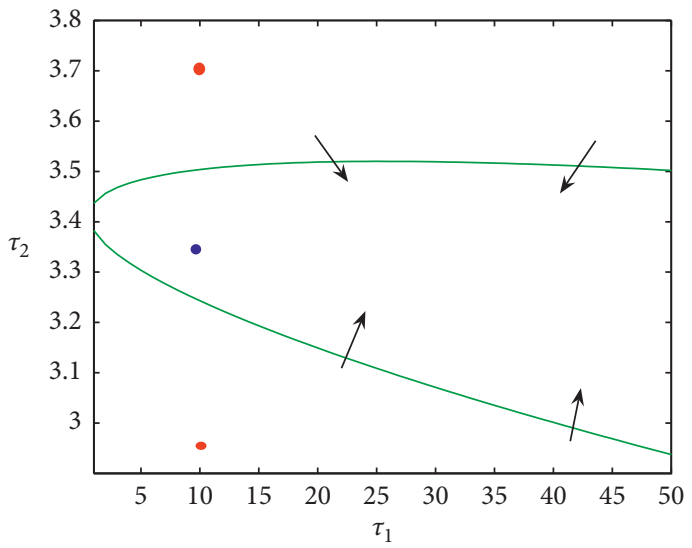

(c)

FIGURE 8: The feedback strength sets: $k_{1}=-1$ and $k_{2}=-2$. The arrow directions point to an unstable region. (a) The crossing set $\Omega=(0.9893$, 2.6715). (b) Stability crossing curves $\tau^{1}$ in $\left(\tau_{1}, \tau_{2}\right)$ plane. (c) The zoom-in T 1 plot.
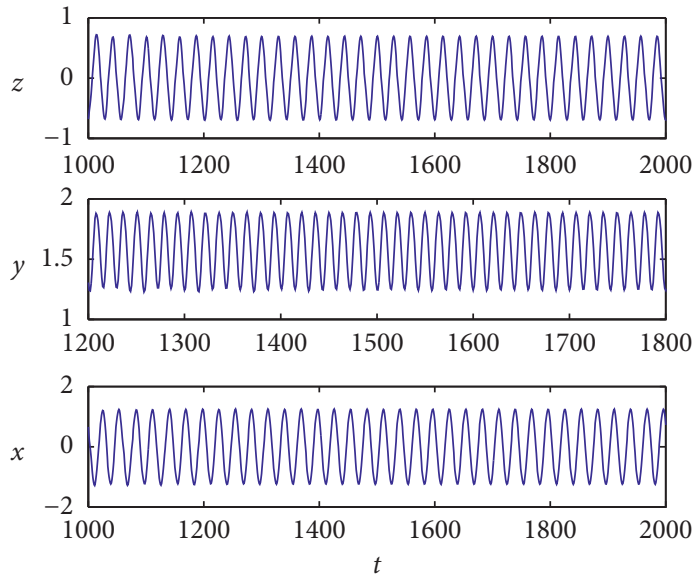

(a)

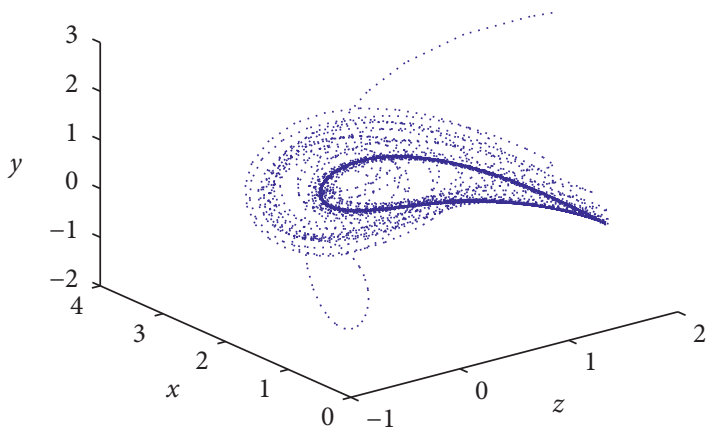

(b)

FIGURE 9: $E_{+}^{*}$ for system (2) is unstable and there exists a stable periodic solution when $\tau_{1}=10$ and $\tau_{2}=0$ with feedback strength sets: $k_{1}=-1$ and $k_{2}=0$. (a) Time series of the solutions. (b) Three-dimensional phase diagram.

(68) can be investigated as system (2) and can also obtain similar results to system (2).

The time-delay feedback controller $k e^{-d \tau}[\mathbf{u}(t)-\mathbf{u}(t-$ $\tau)$ ] with delay correlation coefficients can also be designed to control system (1) which can modify the bifurcation characteristics of a nonlinear system to obtain some specific dynamical behaviors. Note that the strength of feedback control is in the form of $k e^{-d \tau}$, and the function decreases 

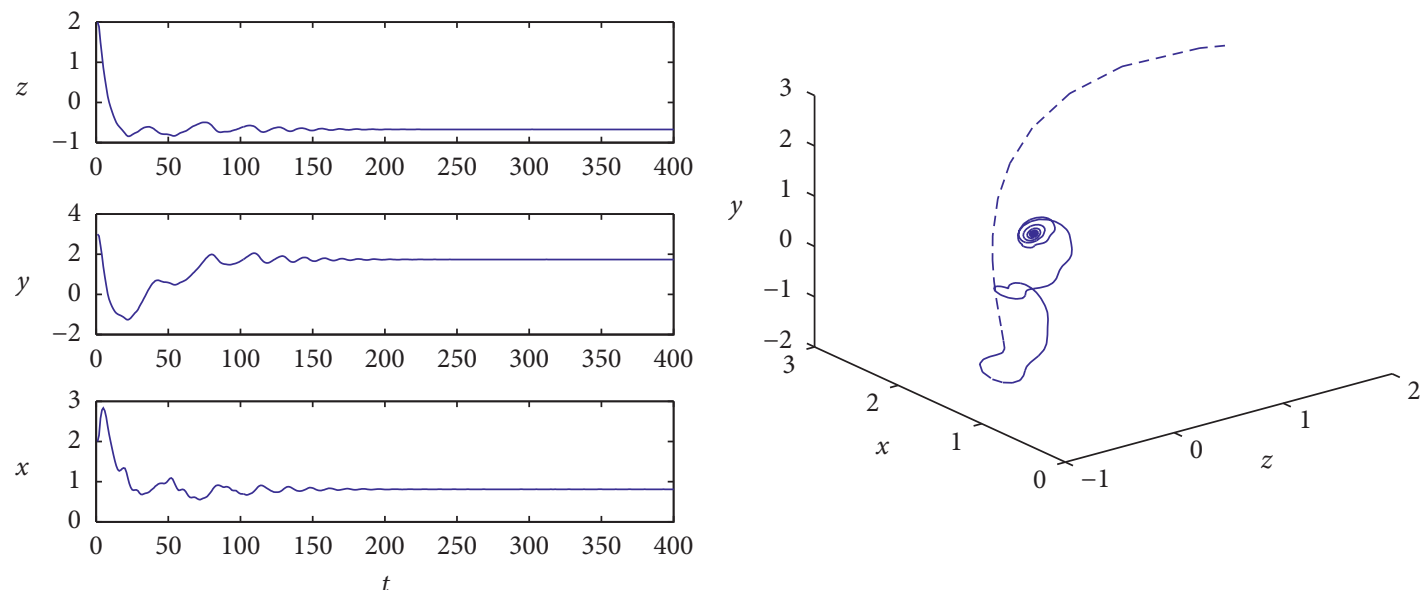

(a)

(b)

Figure 10: $E_{+}^{*}$ for system (2) is stable when $\tau_{1}=10$ and $\tau_{2}=1$ with feedback strength sets: $k_{1}=-1$ and $k_{2}=-2$. (a) Time series of the solutions. (b) Three-dimensional phase diagram.

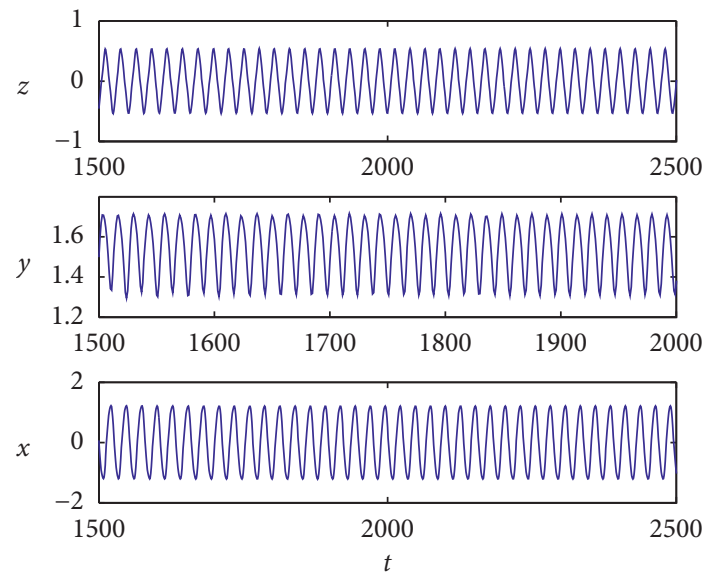

(a)

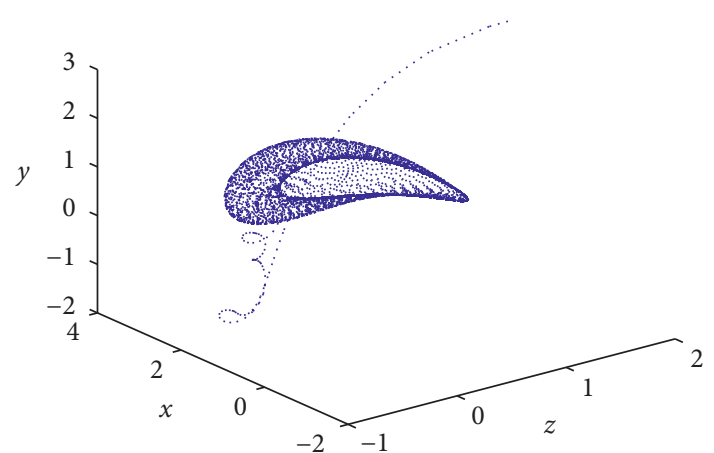

(b)

Figure 11: $E_{+}^{*}$ for system (2) is stable when $\tau_{1}=10$ and $\tau_{2}=3.7$ with feedback strength sets: $k_{1}=-1$ and $k_{2}=-2$. (a) Time series of the solutions. (b) Three-dimensional phase diagram.

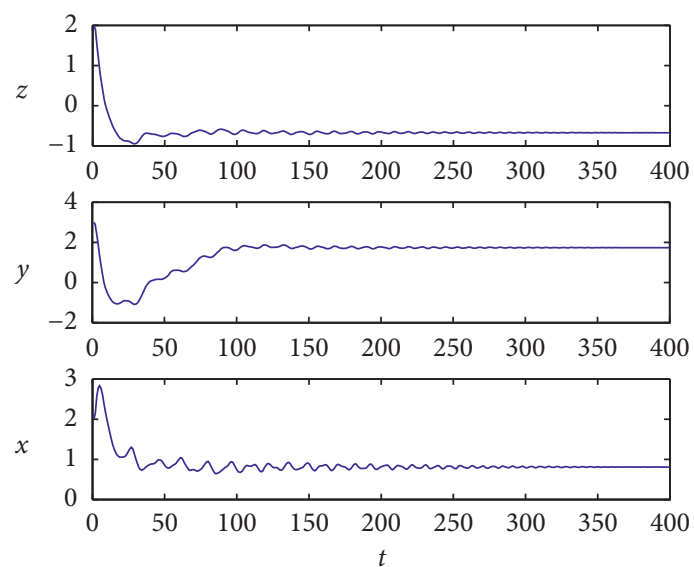

(a)

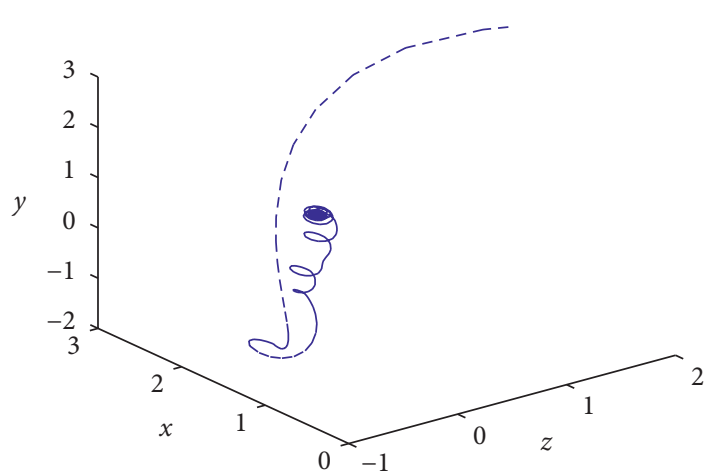

(b)

FiguRE 12: $E^{*}$ for system (2) is unstable and there exists a stable periodic solution when $\tau_{1}=10$ and $\tau_{2}=3.35$ with feedback strength sets: $k_{1}=-1$ and $k_{2}=-2$. (a) Time series of the solutions. (b) Three-dimensional phase diagram. 


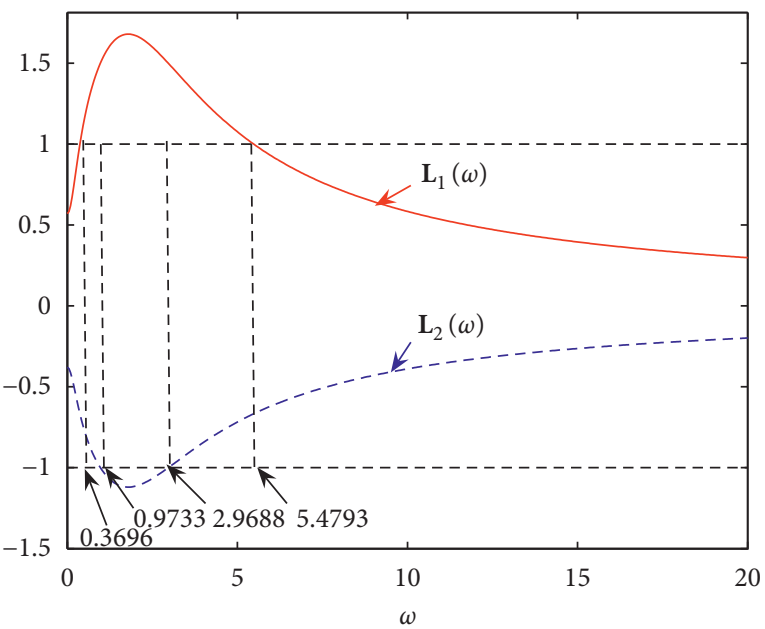

(a)

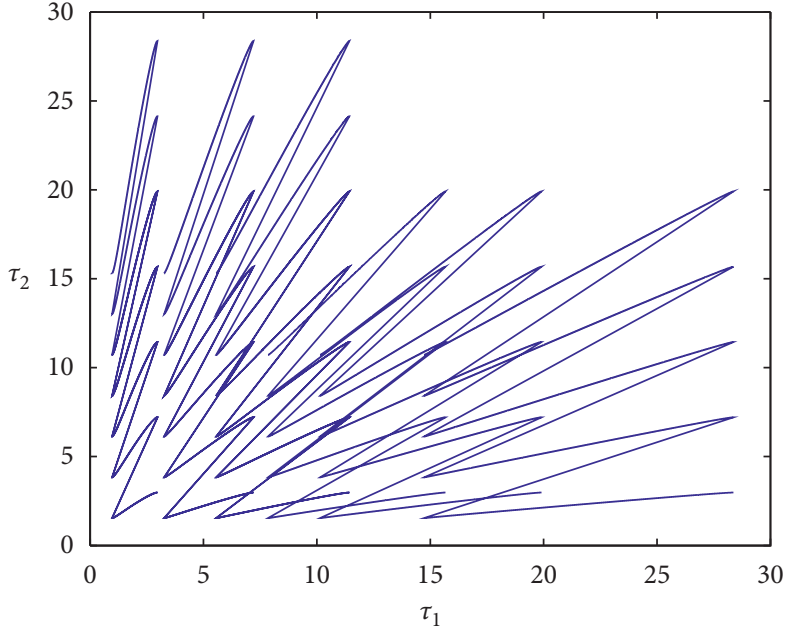

(b)

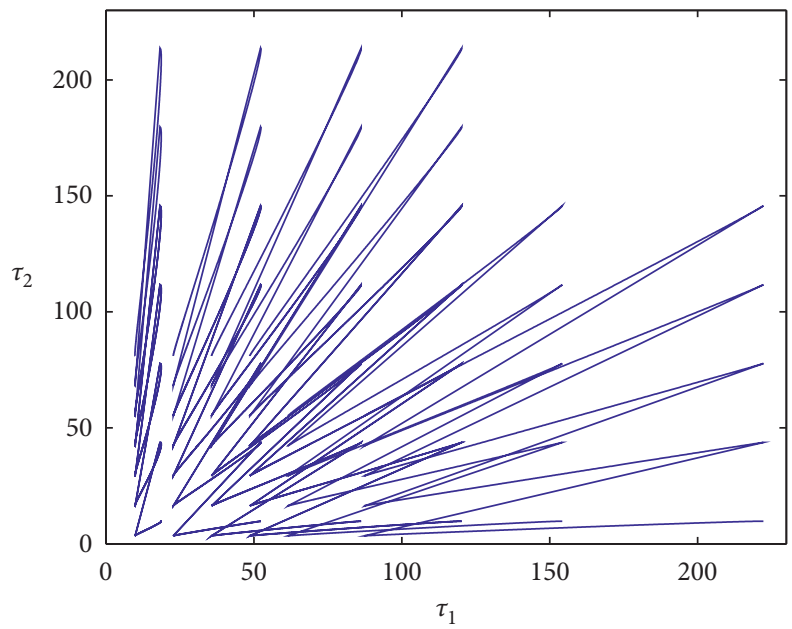

(c)

FiguRE 13: The feedback strength sets: $k_{1}=1$ and $k_{2}=-5$. (a) The crossing set $\Omega=(0.3696,0.9733) \cup(2.9688,5.4793)$. (b) Stability crossing curves $\tau^{1}$ in $\left(\tau_{1}, \tau_{2}\right)$ plane. Stability crossing curves are spiral-like curves along the horizontal axis belonging to type 32 for the crossing set $\Omega_{1}=(0.3696,0.9733)$. (c) Stability crossing curves $\tau^{2}$ in $\left(\tau_{1}, \tau_{2}\right)$ plane. Stability crossing curves are spiral-like curves along the horizontal axis belonging to type 23 for the crossing set $\Omega_{2}=(2.9688 ; 5.4793)$.

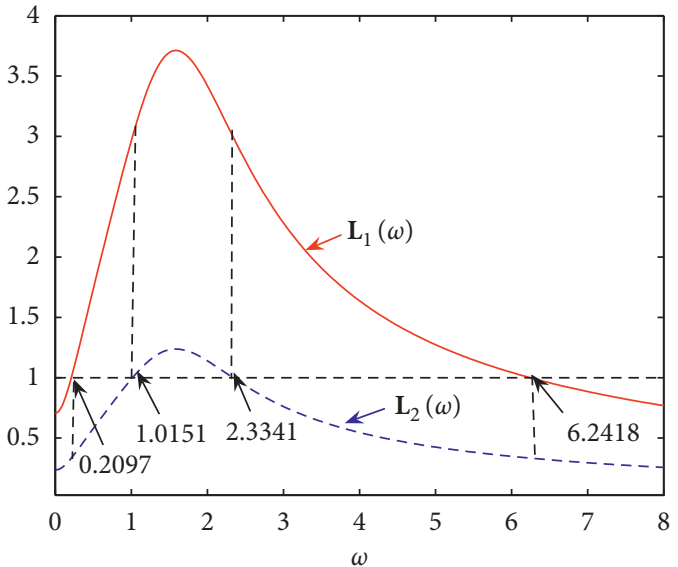

(a)

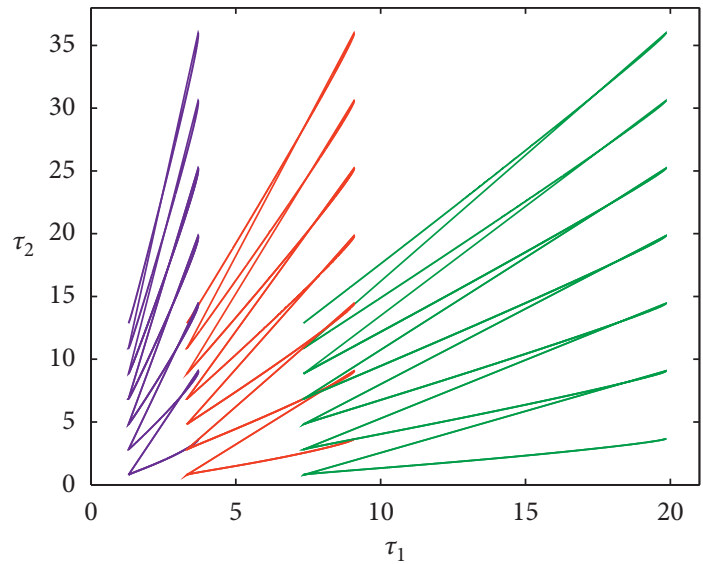

(b)

Figure 14: The feedback strength sets: $k_{1}=-4$ and $k_{2}=2$. (a) The crossing set $\Omega=(0.2097,1.0151) \cup(2.3341,6.2418)$. (b) Stability crossing curves $\tau^{2}$ in $\left(\tau_{1}, \tau_{2}\right)$ plane. Stability crossing curves are spiral-like curves along the vertical axis belonging to type 13 for the crossing set $\Omega_{2}=(2.3341,6.2418)$. 


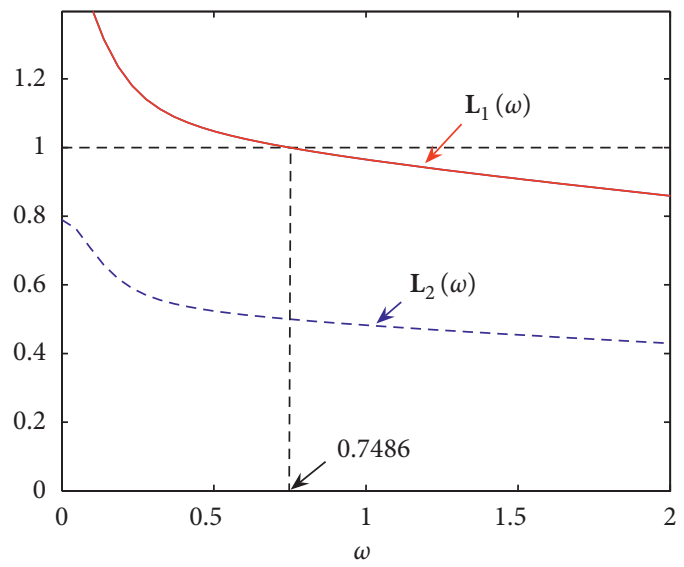

(a)

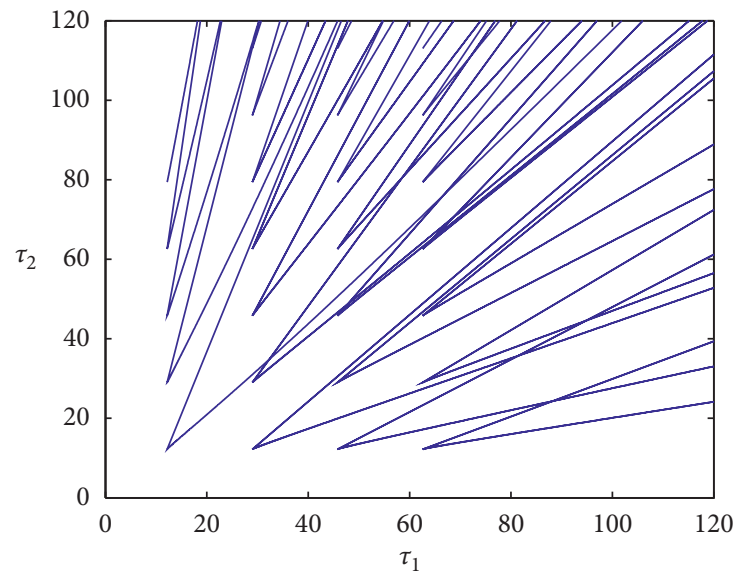

(b)

FIGURE 15: The feedback strength sets: $k_{1}=3$ and $k_{2}=1$. (a) The crossing set $\Omega=(0,0.7486)$. (b) Stability crossing curves $\tau^{1}$ in $\left(\tau_{1}, \tau_{2}\right)$ plane. Stability crossing curves are open-ended curves belonging to type 03 .

exponentially with delay $\tau$. This means that the feedback effects of past states diminish over time. Hence, it can carry out the feedback with time-delay correlation coefficients in system (1). The systems with coefficient dependent delay increase the complexity of the analysis and are challenging, especially those with two time delays. The research is set aside for future consideration.

\section{Conclusion}

This paper analyzes a class of chaotic financial systems with two feedback delays. System (1) exists in chaos under some parameters. The purpose of this study is to control the chaos of the system. For controlling chaos, we improve the DFC method and introduce the double-delay feedback control method in system (1). We introduce the control term in the equation of the interest rate. The system may exist in three equilibria, and we choose one of these equilibria as the research target. It finds that the single delay feedback control can make the system stable and produce the stable switches, i.e., when $\tau_{1}$ changes with $\tau_{2}=0$, system (2) exists stable switches and chaos may disappear. Furthermore, fixing $\tau_{1}$ in a stability interval and taking the delay $\tau_{2}$ as a parameter, proves the existence of the first critical value $\tau_{2}$. At this critical value, the equilibrium loses stability and Hopf bifurcation occurs. The properties of Hopf bifurcation are also studied by using central manifold theory and normal form method for determining the direction of Hopf bifurcation and the stability of bifurcating periodic solution. The abovementioned results are obtained under the condition fixed $\tau_{1}$ in a stability interval; however, if we choose the $\tau_{1}$ in the unstable interval, then there may exist no the critical value $\tau_{2}^{0}$ such that $\tau_{2}^{0}$ is the first Hopf bifurcation value. Hence, for obtaining the complete result separating the stable and unstable regions in the $\left(\tau_{1}, \tau_{2}\right)$, using the stability crossing curve methods in [52], it obtains the curve sets in which Hopf bifurcation occurs in $\left(\tau_{1}, \tau_{2}\right)$ plane for fixed $a, b$, and $c$. By numerical simulations, it can find that the different shape crossing curves, and crossing sets can produce by changing $k_{1}$ and $k_{2}$. Theoretical analysis and numerical simulation results show that, for chaotic financial systems, chaotic oscillation can be controlled by delays. In other words, the multiple delay financial system we study has chaotic oscillations when $\tau_{1}=\tau_{2}=0$. When the delay increases, the chaos disappears, the equilibrium point gains stability or the system appears periodic oscillation, and the periodic solution is generated by the Hopf bifurcation. The DDFC method can control the chaotic behavior of the system more effectively than the DFC method. When $\tau_{1}$ cannot change the chaos behavior of system (2), system (2) can be stabilized by varying $\tau_{2}$ value. These show that the effectiveness of the DDFC method.

\section{Data Availability}

Data sharing is not applicable to this article as all datasets are hypothetical during the current study.

\section{Conflicts of Interest}

The authors declare that there are no conflicts of interest regarding the publication of this paper.

\section{Acknowledgments}

Z. Jiang was supported by National Natural Science Foundation of China (11801014), Natural Science Foundation of Hebei Province (A2018409004), University Discipline Top Talent Selection and Training Program of Hebei Province (SLRC2019020) and Graduate Student Demonstration Course Construction of Hebei Province (KCJSX2020093), 2020 Talent Training Project Funding of Hebei Province. T. Zhang was supported by Shandong Provincial Natural Science Foundation from China (ZR2019MA003).

\section{References}

[1] H. Lorenz, Nonlinear Dynamical Economics and Chaotic Motion, Springer-Verlag, Berlin, Germany, 1989. 
[2] R. Goodwin, Chaotic Economic Dynamics, Oxford University Press, Oxford, UK, 1990.

[3] J. Ma and L. Xie, "The comparison and complex analysis on dual-channel supply chain under different channel power structures and uncertain demand," Nonlinear Dynamics, vol. 83, no. 3, pp. 1379-1393, 2016.

[4] X. Zhan, J. Ma, and W. Re, "Research entropy complexity about the nonlinear dynamic delay game model," Entropy, vol. 19, pp. 1-10, 2017.

[5] J. Ma and L. Sun, "Complexity analysis about nonlinear mixed oligopolies game based on production cooperation," IEEE Transactions on Control Systems Technology, vol. 26, no. 4, pp. 1532-1539, 2017.

[6] F. Si and J. Ma, "Complex dynamics in a triopoly game with multiple delays in the competition of green product level," International Journal of Bifurcation and Chaos, vol. 28, no. 2, Article ID 1850027, 2018.

[7] J. Ma and H. Ren, "Influence of government regulation on the stability of dual-channel recycling model based on customer expectation," Nonlinear Dynamics, vol. 94, no. 3, pp. 17751790, 2018.

[8] B. Xin, W. Peng, and M. Sun, "Optimal coordination strategy for international production planning and pollution abating under cap-and-trade regulations," International Journal of Environmental Research and Public Health, vol. 16, pp. 1-21, 2019.

[9] Z. Wu, G. Hou, and B. Xin, "Has the belt and road initiative brought new opportunities to countries along 205 the routes to participate in global value chains?" SAGE Open, vol. 10, no. 1, p. 2020.

[10] L. Xie, J. Ma, and H. Han, "Implications of stochastic demand and manufacturers' operational mode on retailer's mixed bundling strategy and its complexity analysis," Applied Mathematical Modelling, vol. 55, no. 1, pp. 484-501, 2018.

[11] J. Ma, W. Lou, and Y. Tian, "Bullwhip effect and complexity analysis in a multi-channel supply chain considering price game with discount sensitivity," International Journal of Production Research, vol. 57, pp. 1-21, 2018.

[12] D. Huang and H. Li, Theory and Method of the Nonlinear Economics, Sichuan University Press, Chengdu, China, 1993.

[13] J. Ma, Y. Cui, and L. Liu, "Hopf bifurcation and chaos of financial system on condition of specific combination of parameters," Journal of Systems Science and Complexity, vol. 21, no. 2, pp. 250-259, 2008.

[14] Q. Gao and J. Ma, "Chaos and Hopf bifurcation of a finance system,” Nonlinear Dynamics, vol. 58, no. 1-2, pp. 209-216, 2009.

[15] B. Xin, W. Peng, Y. Kwon, and Y. Liu, "Modeling, discretization, and hyperchaos detection of conformable derivative approach to a financial system with market confidence and ethics risk," Advances in Difference Equations, vol. 2019, no. 1, pp. 1-14, 2019.

[16] J.-h. Ma and Y.-s. Chen, "Study for the bifurcation topological structure and the global complicated character of a kind of non-linear finance system (I)," Applied Mathematics and Mechanics, vol. 22, no. 11, pp. 1240-1251, 2001.

[17] X. Zhang and H. Zhu, "Hopf bifurcation and chaos of a delayed finance system," Complexity, vol. 2019, Article ID 6715036, 18 pages, 2019.

[18] W.-C. Chen, "Dynamics and control of a financial system with time-delayed feedbacks," Chaos, Solitons \& Fractals, vol. 37, no. 4, pp. 1198-1207, 2008.
[19] F. Zhang, G. Yang, Y. Zhang, X. Liao, and G. Zhang, "Qualitative study of a 4D chaos financial system," Complexity, vol. 2018, Article ID 3789873, 5 pages, 2018.

[20] Z. Jiang, Y. Guo, and T. Zhang, "Double delayed feedback control of a nonlinear finance system," Discrete Dynamics in Nature and Society, vol. 2019, Article ID 7254121, 17 pages, 2019.

[21] B. Xin and Y. Qu, "Effects of smart city policies on green total factor productivity: evidence from a quasi-natural experiment in China," International Journal of Environmental Research and Public Health, vol. 16, pp. 1-15, 2019.

[22] G. Kai, W. Zhang, Z. Jin, and C. Wang, "Hopf bifurcation and dynamic analysis of an improved financial system with two delays," Complexity, vol. 2020, Article ID 3734125, 13 pages, 2020.

[23] Z. Wu, G. Hou, and B. Xin, "The causality between participation in GVCs, renewable energy consumption and $\mathrm{CO}_{2}$ emissions," Sustainability, vol. 12, no. 3, p. 1237, 2020.

[24] W. Lou and J. Ma, "Complexity of sales effort and carbon emission reduction effort in a two-parallel household appliance supply chain model," Applied Mathematical Modelling, vol. 64, pp. 398-425, 2018.

[25] J. Ma and B. Bao, "Research on bullwhip effect in energyefficient air conditioning supply chain," Journal of Cleaner Production, vol. 143, pp. 854-865, 2017.

[26] E. Ott, C. Grebogi, and J. A. Yorke, "Controlling chaos," Physical Review Letters, vol. 64, no. 11, pp. 1196-1199, 1990.

[27] L. M. Pecora and T. L. Carroll, "Synchronization in chaotic systems," Physical Review Letters, vol. 64, no. 8, pp. 821-824, 1990.

[28] K. Tanaka, T. Ikeda, and H. O. Wang, "A unified approach to controlling chaos via an LMI-based fuzzy control system design," IEEE Transactions on Circuits and Systems I: Fundamental Theory and Applications, vol. 45, no. 10, pp. 10211040, 1998.

[29] T. Zhang, T. Xu, J. Wang, Y. Song, and Z. Jiang, "Geometrical analysis of a pest management model in food-limited environments with nonlinear impulsive state feedback control," Journal of Applied Analysis \& Computation, vol. 9, no. 6, pp. 2261-2277, 2019.

[30] T. Zhang, N. Gao, T. Wang et al., "Global dynamics of a model for treating microorganisms in sewage by periodically adding microbial flocculants," Mathematical Biosciences and Engineering, vol. 17, no. 1, pp. 179-201, 2020.

[31] H. Zhang and T. Zhang, "The stationary distribution of a microorganism flocculation model with stochastic perturbation," Applied Mathematics Letters, vol. 103, Article ID 106217, 2020.

[32] N. Gao, Y. Song, X. Wang, and J. Liu, "Dynamics of a stochastic SIS epidemic model with nonlinear incidence rates," Advances in Difference Equations, vol. 2019, p. 41, 2019.

[33] G. Liu, H. Qi, Z. Chang, and X. Meng, "Asymptotic stability of a stochastic May mutualism system," Computers \& Mathematics with Applications, vol. 79, no. 3, pp. 735-745, 2020.

[34] M. Rafikov and J. M. Balthazar, "On control and synchronization in chaotic and hyperchaotic systems via linear feedback control," Communications in Nonlinear Science and Numerical Simulation, vol. 13, no. 7, pp. 1246-1255, 2008.

[35] V. Pyragas and K. Pyragas, "Delayed feedback control of the Lorenz system: an analytical treatment at a subcritical Hopf bifurcation," Physical Review E, vol. 73, no. 3, Article ID 036215, 2006.

[36] S. Xu, J. Lam, and Y. Zou, "Delay-dependent approach to stabilization of time-delay chaotic systems via standard and 
delayed feedback controllers," International Journal of Bifurcation and Chaos, vol. 15, no. 4, pp. 1455-1465, 2005.

[37] D.-H. Jiang, J. Wang, X.-Q. Liang, G.-B. Xu, and H.-F. Qi, "Quantum voting scheme based on locally indistinguishable orthogonal product states," International Journal of Theoretical Physics, vol. 59, no. 2, pp. 436-444, 2020.

[38] Z. Jiang, X. Bi, T. Zhang et al., "Global Hopf bifurcation of a delayed phytoplankton-zooplankton system considering toxin producing effect and delay dependent coefficient," Mathematical Biosciences and Engineering, vol. 16, no. 5, pp. 3807-3829, 2019.

[39] Z. Jiang, J. Dai, and T. Zhang, "Bifurcation analysis and control of a delayed plankton ecosystem using delayed feedback control method," International Journal of Bifurcation and Chaos, vol. 30, Article ID 2050039, 2020.

[40] B. Xin, W. Peng, and L. Guerrini, "A continuous time Bertrand duopoly game with fractional delay and conformable derivative: modelling, discretization process, Hopf bifurcation and chaos," Frontiers in Physics, vol. 7, pp. 1-9, 2019.

[41] M. Chi and W. Zhao, "Dynamical analysis of two-microorganism and single nutrient stochastic chemostat model with Monod-Haldane response function," Complexity, vol. 2019, Article ID 8719067, 13 pages, 2019.

[42] T. Zhang, J. Wang, Z. Jiang, and X. Han, "Dynamics analysis of a delayed virus model with two different transmission methods and treatments," Advances in Difference Equations, vol. 2020, p. 1, 2020.

[43] T. Li and W. Zhao, "Periodic solution of a neutral delay Leslie predator-prey model and the effect of random perturbation on the Smith growth model," Complexity, vol. 2020, Article ID 8428269, 15 pages, 2020.

[44] W. Wang, W. Ma, and Z. Feng, "Complex dynamics of a time periodic nonlocal and time-delayed model of reaction-diffusion equations for modeling $\mathrm{CD} 4^{+} \mathrm{T}$ cells decline," Journal of Computational and Applied Mathematics, vol. 367, p. 112430, 2020.

[45] A. Ahlborn and U. Parlitz, "Controlling dynamical systems using multiple delay feedback control," Physical Review E, vol. 72, Article ID 016206, 2005.

[46] K. Pyragas, "Continuous control of chaos by self-controlling feedback," Physics Letters A, vol. 170, no. 6, pp. 421-428, 1992.

[47] K. Pyragas, "Control of chaos via extended delay feedback," Physics Letters A, vol. 206, no. 5-6, pp. 323-330, 1995.

[48] M. Yang and G. Cai, "Chaos control of a non-linear finance system," Journal of Uncertain Systems, vol. 5, pp. 263-270, 2011.

[49] S. Ruan and J. Wei, "On the zeros of a third degree exponential polynomial with applications to a delayed model for the control of testosterone secretion," Mathematical Medicine and Biology, vol. 18, no. 1, pp. 41-52, 2001.

[50] S. Ruan and J. Wei, "On the zeros of transcendental functions with applications to stability of delay differential equations with two delays," Dynamics of Continuous, Discrete and Impulsive Systems, vol. 10, pp. 863-874, 2003.

[51] J. Hale, Theory of Functional Differential Equations, Springer, New York, NY, USA, 1977.

[52] K. Gu, S.-I. Niculescu, and J. Chen, "On stability crossing curves for general systems with two delays," Journal of Mathematical Analysis and Applications, vol. 311, no. 1, pp. 231-253, 2005.

[53] P. Bi and S. Ruan, "Bifurcations in delay differential equations and applications to tumor and immune system interaction models," SIAM Journal on Applied Dynamical Systems, vol. 12, no. 4, pp. 1847-1888, 2013.

[54] B. Hassard, N. Kazarinoff, and Y. Wan, Theory and Application of Hopf Bifurcation, Cambridge University Press, Cambridge, UK, 1981. 